\title{
Nanophononics: state of the art and perspectives
}

Sebastian Volz ${ }^{1}$, Jose Ordonez-Miranda ${ }^{1}$, Andrey Shchepetov ${ }^{2}$, Mika Prunnila ${ }^{2}$, Jouni Ahopelto ${ }^{2}$, Thomas Pezeril ${ }^{3}$, Gwenaelle Vaudel ${ }^{3}$, Vitaly Gusev ${ }^{4}$, Pascal Ruello ${ }^{3}$, Eva M. Weig ${ }^{5}$, Martin Schubert ${ }^{5}$, Mike Hettich ${ }^{5}$,

Martin Grossman ${ }^{5}$, Thomas Dekorsy ${ }^{5}$, Francesc Alzina ${ }^{6}$, Bartlomiej Graczykowski ${ }^{6}$, Emigdio Chavez-Angel ${ }^{6, a}$, J. Sebastian Reparaz ${ }^{6}$, Markus R. Wagner ${ }^{6}$, Clivia M. Sotomayor-Torres ${ }^{6,7}$, Shiyun Xiong $^{8}$, Sanghamitra Neogi $^{8,9}$, and Davide Donadio $8,10,11,12, \mathrm{~b}$

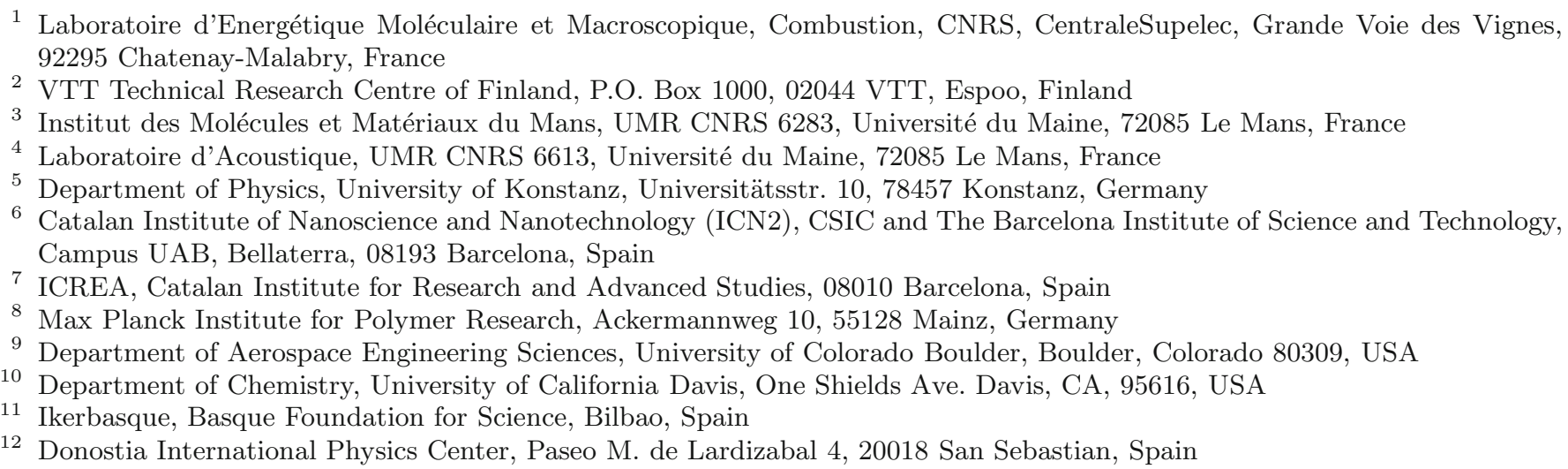

Received 7 September 2015 / Received in final form 3 December 2015

Published online 20 January 2016

(C) The Author(s) 2016. This article is published with open access at Springerlink.com

\begin{abstract}
Understanding and controlling vibrations in condensed matter is emerging as an essential necessity both at fundamental level and for the development of a broad variety of technological applications. Intelligent design of the band structure and transport properties of phonons at the nanoscale and of their interactions with electrons and photons impact the efficiency of nanoelectronic systems and thermoelectric materials, permit the exploration of quantum phenomena with micro- and nanoscale resonators, and provide new tools for spectroscopy and imaging. In this colloquium we assess the state of the art of nanophononics, describing the recent achievements and the open challenges in nanoscale heat transport, coherent phonon generation and exploitation, and in nano- and optomechanics. We also underline the links among the diverse communities involved in the study of nanoscale phonons, pointing out the common goals and opportunities.
\end{abstract}

\section{Introduction}

Phononics is the research field targeting the investigation, control and application of vibrations in solids or liquids that manifest as sound or heat. Phonons are quantized vibrations in condensed phases and obey the laws of quantum mechanics in the same way as other quantum particles, electrons and photons, that are commonly exploited as energy and/or information carriers. In contrast with electrons and photons, for which the scientific discoveries of the past two centuries have been readily converted into technology, only recently different communities have

\footnotetext{
a Present address: Institut für Physik, Johannes Gutenberg Universität Mainz, Staudingerweg 7, 55128 Mainz, Germany.

b e-mail: donadio@mpip-mainz.mpg.de
}

been deploying efforts to achieve control of phonons over a broad range of scales, especially at micro- and nanoscale, hence nanophononics. Such efforts have been stimulated by the ever increasing importance that phonons assume, both per se and interacting with electrons and photons, in a very broad range of technological applications, encompassing nanoelectronics $[1,2]$, renewable energy harvesting [3,4], nano- and opto-mechanics [5], quantum technologies [6,7], and medical therapy, imaging and diagnostics [8]. While applications have been developed by very diverse communities, starting from disparate principles, it is now accepted that further progress stems from an improved understanding of the physics of phonons both in their coherent and incoherent forms.

Fundamental phenomena, such as standing vibrations in mechanical resonators, surface acoustic waves, and heat 
conduction, which are well assessed and described by the classical physics of macroscopic continuum media, have to be reconsidered at the nanoscale, taking into account the effects of confinement and dimensionality on lattice vibrations, the atomistic nature of matter, and the enhanced interaction of phonons with electrons and light. It goes without saying that such wealth of new physical phenomena entails a corresponding amount of challenges and opportunities.

Phonons, as all quantum entities, hold a dual nature as particles and waves, which manifests itself in different conditions. Lattice vibrations can be conveniently decomposed into eigenmodes with the properties of plane waves. The identification of eigenmodes and eigenfrequencies $\omega(q)$ is the fundamental step to describe phonons in harmonic lattice dynamics at any range of frequency, from sound and gigahertz hyper-sound to coherent terahertz waves and heat. The phononic band structure imparts sufficient information to describe not only the propagation of coherent waves, but also the thermal properties of materials, e.g. heat capacity and ballistic conduction. For this reason, major efforts have been deployed to harness phononic band structures by micro and nanostructuring. Lattice vibrations transmit energy as wave packets, that have particle-like behaviour. It is convenient for example to treat phonons as particles to describe heat diffusion, using the Boltzmann transport equation, in which the dissipative terms are introduced as phonon scattering processes. Treating phonons like particles, using concepts such as scattering and phonon mean free paths, provides a natural framework to describe fundamental mechanisms of transport, and to design materials, e.g. phononic crystals $[9,10]$ and hierarchical thermoelectric nanostructures [4].

Phonons are bosons: This means that, in principle, one cannot describe phonon populations using classical equipartition at temperatures lower than the Debye temperature of the materials under consideration, which corresponds to the energy of the mode with the highest frequency expressed in Kelvin $\left(\Theta_{D}=\hbar \omega / k_{B}\right)$. For example, the Debye temperature for silicon based materials is $\sim 600 \mathrm{~K}$ and for carbon nanostructures it can be as high as $1500 \mathrm{~K}$. Consequently, these systems at room temperature are deep in the quantum regime with regard to their thermal properties. In addition, as a consequence of obeying Bose-Einstein's statistics, phonons over a broad range of frequencies are excited at room temperature and are actively involved in transport as either energy or information carriers. Therefore, in contrast with electronic applications, where one targets only the electrons with energy close to the Fermi level, in devising phononic devices, one has to consider the whole population of phonons over a broad range of frequencies. The difficulty of working with a broad spectrum of excitations naturally poses major challenges in designing thermal devices such as cloaks and rectifiers [11].

Different scientific communities in the fields of optics, electronics and materials science, aiming at very diverse goals, have met similar issues related to the very nature of phonons and of their transport properties at the nanoscale. As we will illustrate in the following sections, not only fundamental challenges but also opportunities for applications arise, from phonon confinement in systems with reduced dimensionality, enhanced scattering and phonon modulation from interfaces, establishment of unusual transport regimes at high temperatures, and modified interactions with light and electrons. On the one hand, different research groups involved in nanoscale heat transport, in the generation and use of coherent phonons, and in nano- and opto-mechanics have tackled the above mentioned fundamental issues to various extents, achieving remarkable progress. On the other hand, the exchange of information among the different communities, stemming from very diverse background, has been so far erratic, and it is evident that the field of nanophononics would benefit from an enhanced collaborative crossdisciplinary effort.

In this colloquium paper we review the fundamental physical concepts of nanophononics that stand at the basis of the recent developments achieved in the area of heat transfer, coherent sources, nanomechanics and optomechanics, and, more importantly, we identify a set of challenges that could be met by the synergistic interaction of these diverse communities.

In the next section we review the role of phonons as heat carriers, and how their properties can be tuned by materials engineering at the nanoscale to achieve control on thermal transport. Progresses in probing, generating and exploiting tailored coherent phonon wave packets are reported in Section 3, while challenges and advances in the field of nano- and opto-mechanical resonators are described in Section 4. Potential applications and the perspectives, which will benefit from improved synergy among the diverse nanophononic communities are illustrated in Section 5, while a brief summary and conclusions are given in Section 6 .

\section{Phonons and heat}

\subsection{Heat conduction}

Nanoscale heat conduction issues affect several technological applications including heat-assisted magnetic recording, phase change memories, thermal management in micro- and opto-electronics, thermoelectric energy conversion, thermal energy storage, heating and cooling of buildings and vehicles, manufacturing, and biomedical devices. When the system size reaches the nanoscale, heat conduction is affected by two physical effects: (i) phonon confinement, which modifies the density of states [12,13], and (ii) enhancement of boundary scattering in the ballistic regime [14]. The latter effect appears when the system size is of the same order of magnitude as the phonon mean free paths (MFP), which cover a wide range from a few nanometers to a few microns. In these scenarios, the surface or interface scattering becomes predominant compared to the internal scattering, and many of the thermal phonon studies primarily discuss the various ways of handling boundary scattering [15-18]. System sizes 
comparable to the thermal wavelengths lead to phonon confinement, which in turn results in the creation of standing waves and in reduced phonon transmission, due to both reduction of group velocities and alteration of relaxation times [19]. Low-dimensionality bringing about the complete frustration of specific directions of propagation through confinement and the appearance of noncontinuous mode dispersion is then expected to affect room temperature heat conduction in micro-to-nanoscale devices. These effects combine to the well assessed temperature dependence of heat capacity and thermal conductivity, which is a consequence of the quantum nature of phonons. The strong temperature dependence is evident in various effects: for instance, the phonon mean free paths are inversely proportional to temperature when Umklapp phonon-phonon interactions in non-metals are predominant, i.e. at high temperatures. The prominent phonon wavelength is inversely proportional to temperature as well as predicted by phonon Wien's Law. As a consequence, alteration of heat transport is significant at low temperatures also in macroscopic systems.

The above-mentioned heat transport mechanisms at the nanoscale suffer from limited available theoretical and experimental descriptions. The impact of surface and interface scattering on room temperature heat conduction in sub-micron size systems has been shown in supported films or free-standing membranes, superlattices, porous media and nanowires [20,21]. However, the modeling and detailed understanding of phonon scattering at boundaries and interfaces remain to be established. Indeed, usual acoustic and diffuse mismatch models largely fail in predicting thermal resistances. Confinement or wave coherence effects have been observed in nanowires and films via rather indirect ways [19], while stronger evidences have been obtained in superlattices [22-24]. Modeling phonon properties beyond the harmonic approximation, including accurate relaxation times, has recently progressed owing to the application of density functional theory (DFT) to compute dispersion relations and heat transport [25-27]. Recent self-consistent and variational approaches to solve the Boltzmann transport equation with DFT anharmonic force constants allow us to go beyond the single-mode relaxation time and the local nearto-equilibrium approximations, providing more accurate calculations of the intrinsic thermal conductivity of crystals [28], and deeper insight into the role of collective excitations in non-diffusive heat transport in low-dimensional nanostructures $[29,30]$. However, the use of DFT remains limited to periodic systems consisting of few atoms. In addition, the implementation of first principles approaches is so far restricted to the Boltzmann transport equation, in which anharmonic terms are usually truncated to the third order, and scattering effects related to disorder or broken periodicity are introduced semi-empirically. For example, isotopic and boundary scattering rates are often fitted or introduced ad hoc, as DFT calculations cannot reach the length scale of the surface roughness.

An approach relying on a complementary set of approximations, which allows one to calculate thermal con- ductivity in more complex systems, is based on classical Molecular Dynamics (MD) [31,32]. MD directly provides the atomic trajectories at well defined thermodynamic conditions, and one can compute heat fluxes and local temperatures. Its application extends up to a few millions of atoms, i.e. three-dimensional objects of few hundred nanometers, over several tens of nanoseconds, thus making it possible to probe nanostructures and nanostructured materials. In turn, the reliability of MD simulations depends on the validity of the interatomic empirical potentials, which may turn out to be inaccurate in reproducing the phonon spectrum, especially in the high frequency range. MD relies on the integration of Newton's equation of motion, therefore it does not represent quantum (Bose-Einstein) populations and its prediction is only valid at high temperature. A posteriori quantum corrections have been proven inaccurate [33], and quantum dynamics approaches are also of limited use [34]. The inclusion of quantum effects in MD simulations of thermal transport remains an open methodological challenge.

Complementary to MD, the Landauer approach [35] with phonon Green's function (GF) is also widely used in thermal transport calculations [36-40]. GF was developed originally for the calculation of electron transport [41] and it has been only recently applied to phonon transport $[36,37]$. GF is normally used with harmonic force constant matrices and allows seamless incorporation of the Bose-Einstein statistics for phonons. As a consequence, this approach is particularly suitable to treat ballistic transport at relatively low temperatures. Another advantage of atomistic GF is that it allows one to treat semi-infinite open systems [20]. However, this method can not be used to predict thermal transport at high temperatures where anharmonic effects are important. Anharmonic GF has also been formalized, but, due to its high complexity and computational costs, its use has been so far limited to small systems, such as molecular junctions $[38,39]$. Even when recursive harmonic GF is used, the complexity of the calculation scales with the number of atoms per area like $N^{3}$, thus preventing the applicability of this approach to systems with a cross-section larger than few nanometers. This limitation may be overcome by using the elastic scattering matrix approach [42], implemented with a partitioning-and-knitting algorithm, although applicable only in the case of short-range interactions [43]. Nevertheless the simulation of extended defects and of metal/insulator interfaces is still a formidable task. For example, it remains a challenge to model the fundamental mechanism of the metal/dielectric interfacial resistance, which involves a coupling between electrons and phonons of the two systems. So far, modeling relies on the diffuse mismatch model, which is reasonably accurate if one performs explicit calculations of electron-phonon interactions [44].

Experimental techniques - be it based on the $3 \omega[45,46]$ and the time-domain thermoreflectance techniques [47] or the scanning thermal microscopy [48] - have proven to be robust in estimating the thermal conductivity, thermal conductance and interfacial properties. The need for 
accurate and reliable thermal conductivity measurements has seen sophisticated developments of the $3 \omega$ method [49, $50]$, with a sensitivity of less than $10 \mathrm{nW} /(\mathrm{m} \mathrm{K})( \pm 5 \times$ $10^{-3} \mathrm{nW} /(\mathrm{m} \mathrm{K})$ at room temperature) and a very high resolution $\left(\Delta \kappa / \kappa=10^{-3}\right)$. Likewise, the need to probe buried interfaces, as well as to elucidate the frequency dependence of the thermal conductivity, has nurtured the development of frequency domain thermoreflectance [51] so that the thermal conductivity of buried layers can be determined. A key target of the latter and related methods is to determine the spectral thermal conductivity. Notably, methods like transient thermal gradient [52] have shown evidence of non-diffusive thermal transport over micrometer lengths in $\mathrm{Si}$ at room temperature. Furthermore, among the more recent developments, laser Raman scattering thermometry [53,54], holds a great promise not only to measure thermal conductivity of thin films but also to map temperature fields with sub-micrometer resolution in a contactless fashion and without the use of a metal layer transducer on the material to be examined. While a fully controlled spectroscopic determination of the modal contribution of terahertz phonons to heat transport remains experimentally challenging, progresses have been achieved in the measurement of phonon MFP [55], either by varying the frequency and the width of a pump beam with the maximal frequencies remaining below the terahertz regime or by designing single-mode cavities [56]. Designing a "thermocrystal" [57] by combining scattering and phononic band gaps to decrease thermal conductivity without limit at room temperature therefore poses major realization challenges.

\subsection{Confinement effects in low-dimensional structures: discretisation and deviation from linear behavior}

In a similar way as electrons and photons experience dimensional confinement, phonons too are subjected to discretisation of the density of states and dramatic changes in their dispersion relation. In fact, confinement is an important component of phonon engineering since not only the density of states of confined phonons, but also their frequency and symmetry depend on the geometrical shape of the cavity and the acoustic mismatch between the cavity constituents. This makes confined phonons characteristic strongly dependent on the configuration of the structure. In a homogeneous plate, for example, the acoustic mismatch with the surrounding air leads to the confinement in $1 \mathrm{D}$ of the mechanical energy of the elastic waves supported in the system. In this context, free-standing ultra-thin $\mathrm{Si}$ films are a text-book example to investigate experimentally both size and stress effects on dispersion relations.

The propagation of acoustic waves is often described within the elastic continuum theory, which is usually valid provided the wavelength of elastic waves is significantly larger than the atomic lattice constant. However, the discrete nature of the atomic lattice is ignored. Figure 1a shows the three solutions for the bulk case, which have linear dispersion relations at low wavenumbers. As a result of the spatial continuity being broken in one dimension, together with stress-free boundary conditions, three types (a)

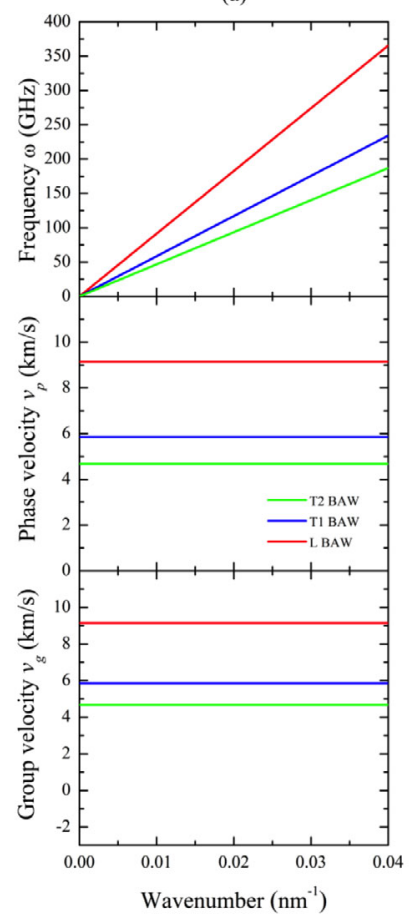

Fig. 1. Dispersion relation of acoustic phonons propagating in (a) [110] of bulk silicon and (b) [110] of 250 nm thick Si (001) membrane.

of plate waves are found as shown in Figure 1b. They are classified according to their displacement about the midplane of the membrane into antisymmetric (A) or flexural, symmetric $(\mathrm{S})$ or dilatational Lamb waves and shear horizontal (SH) waves [58]. Therefore, phonon confinement in membranes is a means to tailor the dispersion relation and, thereby, engineering phonon propagation, with membrane dimension acting as the tuning parameter $[59,60]$. The introduction of controlled stress $(\sigma)$ in the membrane offers an additional degree of freedom to tailor the phonon dispersion relations [61]. A schematic representation of a stressed membrane with a surrounding strain-controlling frame is shown in Figure 2a [62].

Bulk phonon dispersion relations are traditionally determined by neutron scattering, however, the details of the curves are poorly resolved. Thus, suspended membranes have been preferentially studied by Brillouin light scattering (BLS) investigating thermally activated acoustic phonons (acoustic waves) in the $\mathrm{GHz}$ range. A typical configuration of BLS is shown in Figure $2 \mathrm{~b}$ where the angle of incidence of the laser beam incidence is equal to the scattering angle and is denoted by $\theta$. Due to in-plane momentum conservation, the magnitude of the scattering wave vector is given by:

$$
q=\frac{4 \pi}{\lambda} \sin \theta
$$

which means that by varying the angle of incidence, data points are obtained for different wave vectors close to $q=0$. For example, varying the scattering angle $\theta$ in the range $11^{\circ}-50^{\circ}$, corresponds approximately, according 

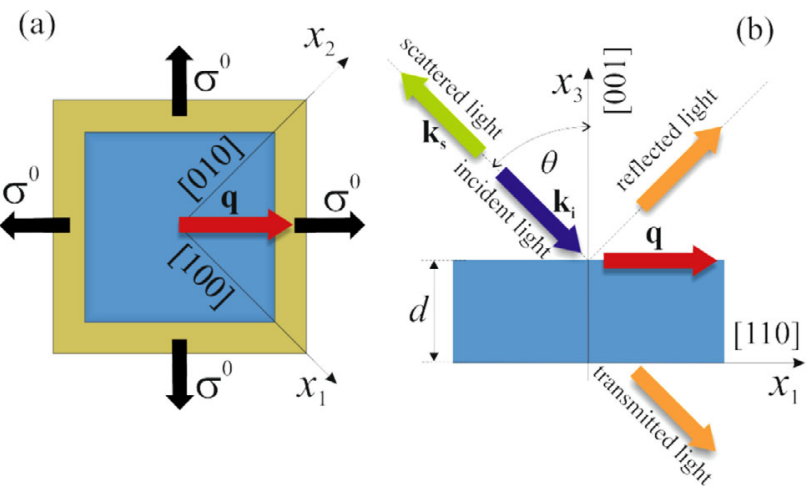

Fig. 2. Schematic illustration of (a) top view-relative orientation of sample and biaxial stress, (b) lateral view-Brillouin light scattering geometry. Symbol $d$ stands for the membrane thickness, $\sigma^{0}$ is the component of the stress tensor $\sigma_{i j} . k_{i}$, $k_{s}$ and $q$ denote incident light, scattered light and scattering wavevectors respectively, $\theta$ is the scattering angle. Vectors in the schematic are not to scale. (a)

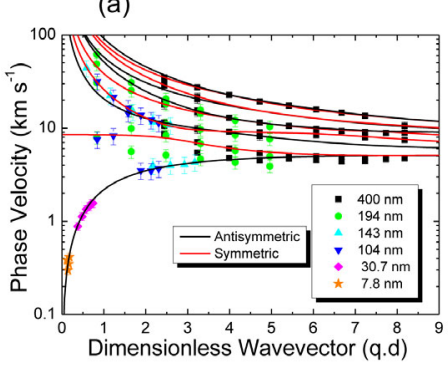

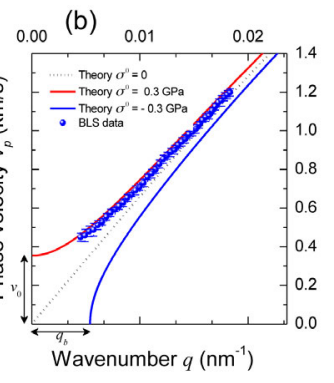

Fig. 3. (a) Experimental and calculated phonon phase velocity of membranes with thicknesses, $d$, from $400 \mathrm{~nm}$ down to $\sim 8 \mathrm{~nm}$. (b) Experimental and calculated phase velocity obtained for A0 mode propagating in $27 \mathrm{~nm}$ thick membrane under an applied load $\sigma^{0}=0.3 \mathrm{GPa}$.

to equation (1), to the range of wavenumbers $q$ from 0.00451 to $0.0180 \mathrm{~cm}^{-1}$. The dispersion relations over the whole Brillouin zone can be obtained by synchrotron $\mathrm{X}$-ray thermal diffuse scattering [63]. However, it is early days to assess the real advantages of this technique unless it is combined with a strong theoretical analysis for an improved overall spatial resolution to avoid unnecessary inhomogeneous broadening.

Figure 3a displays the measured dispersion relations of membranes with $\sigma^{0}=0$ and thickness $d$ ranging from $\sim 8$ to $400 \mathrm{~nm}$. The phase velocity against the dimensionless wavevector $q \cdot d$ is plotted in a logarithmic scale to emphasize the dramatic decrease of the zero-order flexural mode (A0) velocity in ultra-thin membranes. The A0 mode is highly dispersive in the range where its wavelength is larger than the membrane thickness and its velocity decreases down to $300 \mathrm{~m} / \mathrm{s}$ in the $8 \mathrm{~nm}$ membrane. On the contrary, the velocity converges to the surface wave velocity in the [110] direction of bulk silicon of $5085 \mathrm{~m} / \mathrm{s}$ for thicker membranes. In the highly dispersive regime both $v_{p}$ and $v_{g}$ are directly proportional to $q$, i.e., $\omega(q)$ is a quadratic function.
There are several outstanding scientific challenges, with respect to phonon modes and their dispersion, including experimental methods to determine the polarisation of the heat carrying phonons and the impact of the dynamic modal occupation on anharmonicity and, thereby, normal scattering. In particular, anharmonic decay is likely to affect the time evolution of the MFP spectral contribution to the thermal conductivity, although a priori it is not clear if in a strong or weak manner. While phonon polarisation is relevant to differentiate phonon states and would impact diode-like functions and information processing by phonons, the time-dependent mode occupation would help to understand non-linear processes and their impact on the MFP spectral contribution to the thermal conductivity. Recent developments in methods for dynamic studies simultaneous with structural ones, such as time-resolved thermal diffusive scattering [64], may provide a muchneeded insight into anharmonicity-dependent properties.

When tensile or compressive stresses are built-in the dispersion relation of the A0 mode changes remarkably. Figure $3 \mathrm{~b}$ presents dispersion curves calculated for a $27 \mathrm{~nm}$ thick membrane for a range of accessible wavenumbers. For $\sigma^{0}=0$ the phase velocity is directly proportional to the wavenumber, as discussed previously. On the contrary, under tensile or compressive stresses the dispersion relation becomes non-linear. The calculated curve for the tensile case, which shows a very good agreement with the measured dispersion relation, reveals that $v_{p}$ attains a value $v(q=0)$ different than 0 , as $q$ tends to 0 , i.e., $\omega(q)$ changes from quadratic to linear. Furthermore, the dispersion relation for compressive stress displays ranges of forbidden wavenumbers, where the velocity and frequency of A0 acquire imaginary values. This behaviour is related to buckling instability due to compressive stress.

\subsection{Phononic metamaterials and surface scattering}

In a pioneering work for materials engineering, Sheng and coworkers proposed the idea of combining standard materials with different elastic moduli so to create a composite with specifically designed acoustic resonances, which break the conventional mass-density law of sound [65]. Such system is dubbed an acoustic metamaterial. The same basic concept of using resonances to modify phonon dispersion and propagation has been recently put forward to modulate hypersound in the gigahertz regime [66] and to limit heat diffusion in the terahertz regime [67].

In phononic crystals, to tune the properties of acoustic phonons with higher frequencies, such as heat carriers at room temperatures, which are well within the terahertz regime, one has to introduce features that match the scale of the relevant phonon wavelengths. Assuming linear dispersion relations, and typical speed of sound in solids of the order of $\sim 10^{3}-10^{4} \mathrm{~m} / \mathrm{s}$, it turns out that one has to deal with heat carriers with nanometer wavelengths, which may be effectively controlled by engineering materials at the nanoscale. On the contrary, the metamaterial approach applies in principles to the frequency domain, thus enabling in principles tuning of phonons which match 


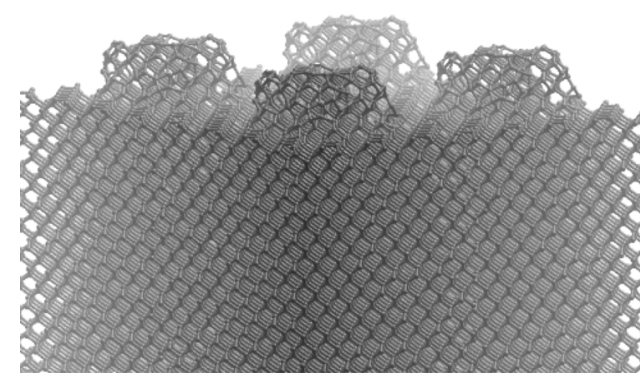

Fig. 4. A model of a silicon thin film with nano-pillars at its surface, proposed as a thermal metamaterial [67].

the frequency of resonant structures, regardless of the relative size scales.

Silicon thin films and suspended membranes provide a promising platform to develop phononic crystals $[10,68,69]$ and metamaterials, as they incorporate the effect of phonon confinement, discussed above, combined with a large surface to bulk ratio. The latter feature makes heat transport extremely sensitive to surface nanostructuring, be it natural roughness generated by the spontaneous growth of native oxide [70] or carefully designed surface nano patterning [67] (Fig. 4). In both cases, the requirement of periodicity, which is necessary for band engineering in phononic crystals, is relaxed, since the reduction of thermal conductivity should stem from localized resonances, in the analogous fashion as the behavior of the original macroscopic sonic crystals as total reflectors for $\mathrm{MHz}$ sound waves. In addition, working with reduced dimensionality systems, such as membranes and nanowires, permits a novel approach to control either gigahertz phonons or heat flow, which consists in adding material on the surface, instead of removing it or introducing obstacles, such as grain boundaries on various scales [71]. In fact, surface resonances from nano pillars were predicted to reduce the thermal conductivity of silicon thin films by about a factor of two with respect to that of a film with flat surface [67]. In turn, experiments and simulations demonstrated a reduction of thermal conductivity in ultra thin suspended membranes of about a factor 30 with respect to the bulk, determined by scattering of phonons by the native oxide at the surfaces [70]. This approach is very promising to design thermoelectric nanostructures, in which thermal transport is inhibited by surface resonances while electrons can flow undisturbed, thus maximizing the conversion efficiency of temperature gradients into voltage. Furthermore, metamaterials allow a greater control of phonon band structure and transport, thus boosting developments of several other fields, in which high frequency phonons play a crucial role, such as sensing, phonon information, thermal rectification or thermal cloaking $[72,73]$.

The concept of designing metamaterials is still in exploratory phase, especially concerning thermal phonon engineering, and several challenges need to be overcome, from theory to fabrication and measurement. At the most fundamental level, besides the issues related to describing phonon scattering at surfaces, it is not yet clear whether the natural incoherence of thermal phonons in the diffusive regime would make resonances ineffective. Furthermore, it is difficult to envisage how structures with a specific size and resonance frequency may be used to efficiently control thermal phonons, considering that in materials like silicon thermal phonons span over a broad range of frequencies and wavelengths and several orders of magnitude of MFP. For example, the introduction of short range disorder in $\mathrm{Si}$ membrane-based 2D phononic crystals was recently shown to modify the phonon properties (dispersion, coherence) in the hypersonic $(\mathrm{GHz})$ range without affecting the room temperature thermal conductivity [74].

Colloidal crystals and polymer composites offer a flexible option to implement phononic crystals and metamaterials. Arranging polymer-decorated colloidal particles in periodic lattices permits the realization of two- or three-dimensional phononic crystals, in which the dispersion relations of hypersonic acoustic modes can be controlled by tuning the features of the polymer linkers and the particle-polymer interface $[75,76]$. This approach introduces further degrees of freedom on top of the nanostructure length scales, commonly employed to engineer phonons in hard materials.

Recent theoretical and experimental efforts in the direction of acoustic metamaterials [77] combined with high level calculations of two- and three-dimensional phononic structures [78] bring closer the long-term aim of mimicking transformation optics with acoustic phonons, so to implement transformation acoustics [79]. Progress in metamaterials would allow the control of sound and heat focusing, and a myriad of related refractive phenomena, enabling broader and more effective use of coherent phonons, as discussed in Section 3.

\subsection{Near field effects}

When the gap between two objects is smaller than the characteristic photon wavelength, near-field radiation starts to predominate heat transfer. Both theoretical $[80,81]$ and experimental [82-84] works have demonstrated that heat transfer through near-field radiation between two objects can be several orders of magnitude larger than the blackbody limit over a limited range of frequency. The electromagnetic field of thermal radiation is composed of propagating and evanescent modes. Evanescent modes contribute to heat transfer by a tunneling mechanism and are the source of near-field radiation. This requires that a dissipative body is brought within the evanescent wave field of a thermal source (subwavelength distance) [85]. Although experiments have been conducted to measure near-field radiation, most of them are in the region with gaps of micrometers and only few of them are dealing with the near-field in nanoscale gaps because of the difficulties in carrying out such experiments. Narayanaswamy et al. $[82,83]$ performed the measurement of the radiative heat flux between two objects separated by a nanoscale gap distance. Their results led them to conclude that the proximity force approximation is not valid for near-field radiation heat transfer. However, in contrast with their conclusions, Rousseau et al. [84] measured the 


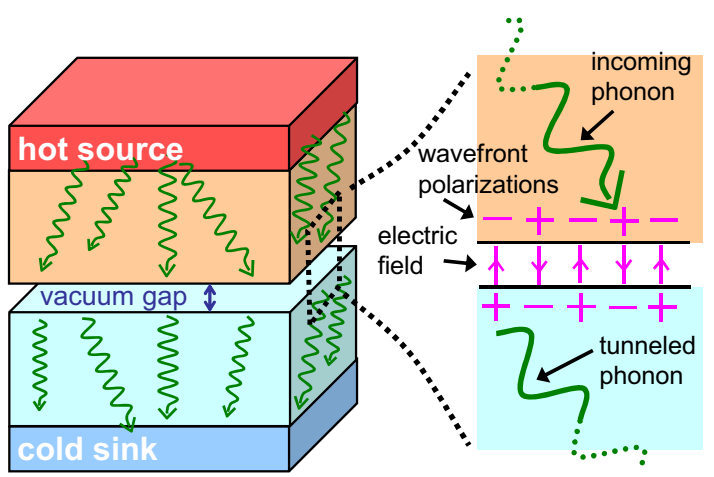

Fig. 5. Illustration of the proposed acoustic phonon heat transfer/tunneling effect through a vacuum gap. A hot source radiates phonons towards a cold sink and vice versa (ballistic case). Single phonons carry an electric field, illustrated by + or - signs of the wavefront polarization. The polarization induces an electric field into the vacuum gap and the field enables finite transmission over the gap [86].

heat transfer in the nanoscale and the results confirmed the proximity approximation. As a result, near-field transfer at nanoscale gaps is still under debate.

Near-field radiation with the dipole or multipole approximation has been extensively investigated on a theoretical basis. Nonetheless, mechanisms taking place at separation distances less than $10 \mathrm{~nm}$ remain unclear. Moreover, this region involves the less understood phonon tunneling effect and transition from the near-field radiation to heat conduction. The first study on this transition to heat conduction was conducted between two nanoparticles by Domingues et al. [87]. By means of molecular dynamics simulations, a transition region with a maximum of thermal conductance was found right before the two nanoparticles are in contact. Acoustic phonons have been identified as viable heat carriers through vacuum gaps in polarizable materials. Polarization induced by phonons produces a macroscopic electric field that enables interbody coupling, as formulated in reference [86] (Fig. 5). Such direct heat conduction through vacuum, referred to also as acoustic phonon tunneling, may occur, for example, between piezoelectric bodies. Very recently, phonon transport across nanoscale gaps has been revisited by means of the phonon GF method $[88,89]$. Within the investigated gap distances, three typical regions with different heat transfer behaviors were identified, i.e., the chemical bond interaction region, the surface charge-charge interaction region, and the dipole-dipole interaction region. These works highlighted the importance of acoustic phonon tunneling in heat transport in the near-field. On the experimental side massive enhancements in the nearfield heat transfer on nanometer scale from scanning probe tip to a substrate have been linked to phonon tunneling effects $[90,91]$. However, at the moment there is no phonon tunneling theory that covers the range of distances and geometries of these experiments.

In polar materials, the evanescent mode is generated by the oscillations of transverse optical phonons (surface phonon polaritons, SPhP). SPhP are hybrid electromagnetic waves that are the result of the coupling between photons and phonons at the interface between two different media [92-94]. Over the past few years, significant research efforts have been devoted to the study of these surface waves, due to their potential applications to improve the thermal performance and stability of nanoscale devices in electronics [95-100]. Taking into account that the surface effects predominate over the volumetric ones due to the high surface area/volume ratio in nanomaterials, the energy transport by SPhP is expected to be particularly important in nano-sized amorphous media, the bulk thermal conductivity of which is usually low and decreases as the size reaches the nanoscale [101-103]. Despite their importance, the contribution of the SPhP to the thermal conductivity of these materials is not well understood to date, especially at nanoscale.

It is well-known that $\mathrm{SPhP}$ can have long propagation lengths, particularly on thin films [98,100,104], which in turn could lead to efficient energy transport. Many research groups have suggested that the $\mathrm{SPhP}$ could be used to enhance the radiative heat transfer in the direction perpendicular to the two parallel surfaces as well as along the parallel surfaces $[84,87,97,99,105]$. Furthermore, Mulet et al. [97] and Narayanaswamy and Chen [106] have shown that in materials that support electromagnetic surface waves, such as silicon carbide or silicon dioxide (glass), it is possible to see not only an enhancement but also a narrowband characteristic of the thermal energy transfer between the two parallel surfaces or between a small particle and a plane surface. By calculating the SPhP contribution to the in-plane thermal conductivity of a thin film, Chen et al. $[98,100]$ showed that the SPhP can increase the thermal energy transport across the thin films as well as in the in-plane direction. These authors reported a thermal conductivity of $4 \mathrm{~W} /(\mathrm{m} \mathrm{K})$ for a $40 \mathrm{~nm}$-thick thin film of silicon dioxide at $500 \mathrm{~K}$, which represents an increase of about $100 \%$ over its bulk phonon thermal conductivity. These last results were based on the kinetic theory and have been confirmed by means of a fluctuation-dissipation approach [107-109]. Recent works have shown promising results on thin films of amorphous polar materials and on how the material size and shape affect the propagation length and SPhP thermal conductivity [110]. While theoretical estimations of thermal fluxes carried by SPhP in thin films have been clearly established revealing their predominance over the conventional vibrational heat channel at characteristic sizes smaller than $300 \mathrm{~nm}$, the experimental demonstration of the existence of the SPhP heat channel remains an open challenge.

\section{Coherent phonons}

Traditional vibrational optical spectroscopic methods (Brillouin, Raman, infrared spectroscopies and inelastic neutron scattering) as well as heat transport properties described in the previous section provide detailed insights in the lattice dynamics in the harmonic and anharmonic regime. In these techniques the phonon population under study is controlled by the temperature of the system, and 
no well-defined phase relation exists among phonons. As pointed out in Sections 2.2 and 2.3, the thermal phonon spectrum can be tailored by means of phononic crystals and/or metamaterials to target specific properties. Another way to study the phonon properties and to envision new applications, is to generate selectively these phonons with external stimuli. Among possible stimuli (electric bias, magnetic field, light), short light pulses offer unique possibilities due to their intrinsic broadband spectrum achievable with femtosecond lasers. The advent of ultrashort laser pulse technologies in the 80's has provided indeed important new tools for the study of phonon dynamics: having access to the time scale of a vibrational period of phonons, phonon wavepackets can be tailored and the phase of phonons can be locked by the ultrafast optical excitation of the lattice degrees of freedom. These new spectroscopic tools and the development of pump-probe methods has led to the field of coherent phonons [111]. It is important to distinguish these coherent phonons from ballistic heat pulses that can be generated and studied at low temperatures [112]. The experimental investigations of coherent phonon dynamics are mainly based on pumpprobe schemes where a femtosecond pump pulse excites an elementary or collective excitations in the system. A second laser pulse delayed in time with respect to the exciting laser pulse $[111,113]$, probes the dynamics of the system as it is brought out of equilibrium by the light pulse and relaxes back. It should be noticed that more recently, the out-of-equilibrium dynamics in solids can be also probed with pulsed X-rays [114] and electron scattering [115]. Different particles, quasiparticles or collective excitations (such as electrons, phonons, polaritons, spins, plasmons, etc.) and their mutual interactions have been studied by time-domain spectroscopy. In this section we focus on the phonons dynamics. Depending on the femtosecond laser interaction with matter, some specific phonons (with well defined $q$ vector, frequency and polarization) can be photogenerated. This field has opened a new playground for the time-domain investigation of electron-phonon coupling, phonon-phonon interactions, and with extension towards the spin-phonon coupling. Moreover, coherent phonons wavepacket, i.e. coherent phonons sources, can be controlled and these phonons have been used to probe solids and artificial nanostructures.

\subsection{Coherent phonon sources: electron-photon-phonon interactions}

Electron-phonon coupling has been investigated in great detail by traditional electronic transport properties and with modulation spectroscopy [116]. In both cases, the phonons and electrons are described through the BoseEinstein and the Fermi-Dirac distribution functions, respectively. With a femtosecond laser pulse different electron-phonon and photon-phonon interaction mechanisms, either an electronic interband transition (above band gap excitation) or a virtual state (below band gap interaction) can be activated depending on the light-matter interaction involved. For the first case, deformation potential interaction, often discussed within the picture of displacive excitation of coherent phonons (DECP), thermoelasticity and inverse piezoelectric effect are of relevance. For the second, electrostriction, often discussed within the picture of stimulated Brillouin-Raman scattering, and magnetostriction are the most important processes, for further details, see reviews about optical phonons [117-119] and acoustic phonons [111,112,120].

With above-band-gap excitations in semiconductors, it is possible to control the population of electrons and holes, which in turn leads to the dynamics determined by electron-phonon coupling. Usually, several different electron-phonon coupling mechanisms have the same time scale. However, depending on the details of the optical interband transition induced by the femtosecond pump laser, on the concentration of photoexcited electron-hole pairs, and on the crystallographic orientation of the photoexcited solids, it is possible to enhance one mechanism with respect to the others. In metals, the coherent optical phonons are usually photoinduced by the deformation potential mechanism, i.e. a modification of the internal interatomic interaction by a rearrangement of the electronic distribution [117]. This disturbance of the electronic distribution has typically the same symmetry as the lattice itself. Hence the deformation potential interaction leads typically to the impulsive excitation of lattice modes reflecting the lattice symmetry, e.g. $A_{1 g}$ modes in Bi. These modes exhibit in general Raman activity [117]. The change of the equilibrium positions of the atoms induced by deformation potential exists as long as the excited electrons and holes do not recover their initial spatial distribution. This mechanism is also responsible for the generation of coherent acoustic phonons $[121,122]$. In the case of metals, due to the very short lifetime of hot electrons, typically below 1 ps [123], only very high frequencies of coherent acoustic phonons (i.e. $\omega>500 \mathrm{GHz}$ ) are generated. These modes are rapidly damped through anharmonic phononphonon and defect collisions, which make their observation experimentally very difficult. In metals, the major part of the excess energy of hot electrons is rapidly transferred to the lattice through intraband relaxation, i.e. electron relaxation through the emission of optical and/or acoustic phonons. The phonons generated via this path have no phase relation, hence the incoherent phonon population increases. Consequently, due to the anharmonicity of the lattice, this effect leads to a rapid lattice expansion or thermoelasticity. This process is usually the main mechanism for the generation of coherent acoustic phonons in metals $[111,112]$. The volume of the material, where this lattice heating takes place, is sometimes larger than the volume given by the absorption length of the optical pump pulse due to hot electron diffusion [124-127]. The ultrafast lattice heating generates both longitudinal and transverse acoustic phonons providing an appropriate symmetry breaking in the crystal for the generation of transverse modes $[128,129]$.

The situation in metals has to be distinguished from that in semiconductors: here the photoexcited electron and hole lifetime can be much longer than 1 ps, which permits to maintain the electronic pressure, 


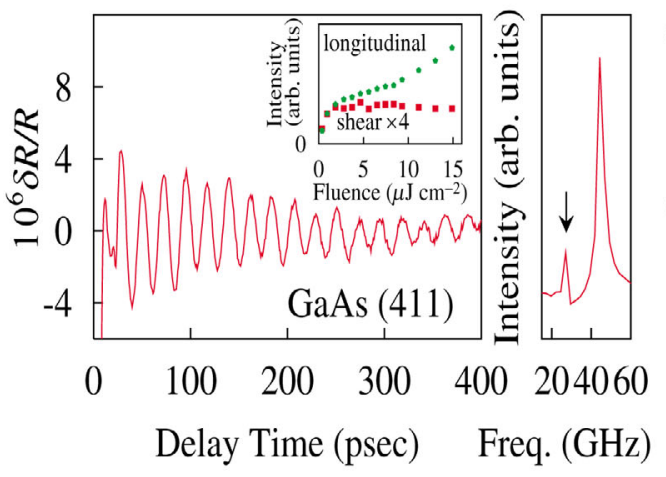

(a)

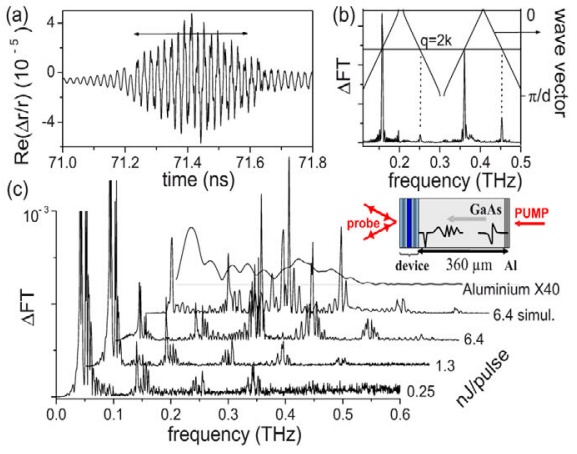

(b)

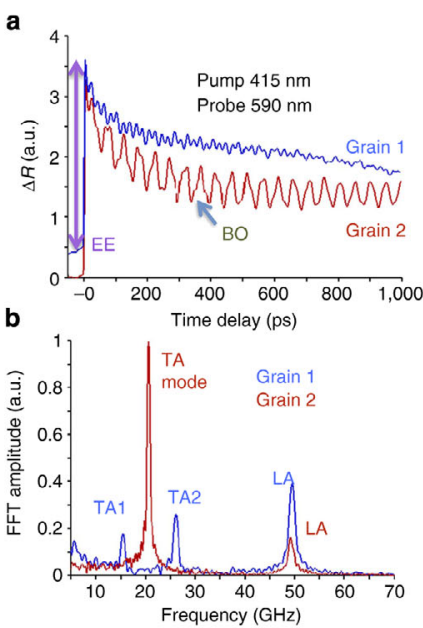

(c)

Fig. 6. (a) Photogeneration of GHz coherent phonon in low symmetry GaAs semiconductor wafer, where at low fluence non linear pump power dependence of LA and TA mode amplitude is a signature of the inverse piezoelectric mechanism. Because of photoinduced saturation of internal electric field, it becomes negligible at high fluence, where the deformation potential prevails for LA modes, while it is inefficient for TA modes [128]. (b) A broadband coherent acoustic pulse is photogenerated in a Al film on the front side. The acoustic phonons propagate through the entire sample and are detected on the back side in the GaAs-GaAlAs superlattice. The detection is achieved through a Brillouin process (Brillouin zone point $q=2 k$ ) that reveals high frequencies up to the $\mathrm{THz}$ regime [130]. (c) Generation of $\mathrm{LA}$ and $\mathrm{TA}$ mode in $\mathrm{BiFeO}_{3}$ ferroelectric materials driven by photoinduced inverse piezoelectric effect based on the photoinduced screening of the internal ferroelectric polarization [131]. The experiments were conducted on various grains in polycristalline sample. Depending on the grain orientation (i.e. on the internal ferroelectric polarization), the efficiency of the generation of LA or TA varies accordingly to the light-induced inverse-piezoelectric effect.

i.e. the deformation potential stress, over a longer time scale. As a matter of fact, the deformation potential mechanism is usually the prevailing mechanism in many semiconductors like GaAs, Si, and Ge for phonons generated in the 1-500 GHz frequency range [132-134]. A special situation arises when the photoexcited carriers are created with pump photons having a quantum of energy equal to the band gap. In that case, the electronic excess energy is zero and no intraband relaxation process occurs. Since no incoherent phonon emission occurs the lattice heating is absent. The details of the dynamics can be very intriguing: a most striking effect of the electronic pressure is the light-induced contraction of the material when illuminated with a laser. This effect stems from a positive photoinduced deformation potential stress, which is in contrast to the negative thermoelastic stress, as heating usually leads to a lattice expansion. The effect of light-induced contraction has been demonstrated for Si [135]. This prevailing contribution of the deformation potential is possible only if no rapid radiative or non-radiative recombination of carriers occurs $[112,120]$. In piezoelectric semiconductors, the inverse piezoelectric effect can be the prevailing mechanism, mainly because a static built-in electric field (surface space-charge electric field, $\mathrm{p}-\mathrm{n}$ or Schottky junction electric field) of the order of $>100 \mathrm{kV} / \mathrm{cm}$ may exist. This static electric field leads to strain in the material, i.e. stored energy, which relaxes upon excitation of electronhole pairs that screen this field on ultrashort time scales. This mechanism has been shown to be efficient for the generation of optical phonons in GaAs by transient depletion field screening (TDFS) $[136,137]$. This mechanism leads then to a displacement of the equilibrium position of cations, which is consequently responsible for a collective lattice motion. The optically excited carriers are separated by the built-in electric field, so that the screening occurs over a distance larger than the unit cell and usually also larger than the light penetration depth. If the normal of the irradiated surface is a piezoactive crystallographic direction, it can also lead to a macroscopic inverse piezoelectric effect that induces a macroscopic change of the lattice volume (contraction or expansion) with an emission of coherent acoustic phonons [128,138,139]. Over a given pump fluence it is even possible to fully screen the built-in field and to reach a saturation regime of the phonon emission as shown in Figure 6a for the TA mode. The LA mode does not show saturation, since at high fluence the deformation potential mechanism becomes the prevailing mechanism in comparison to the piezoelectric effect. Based on these experimental results, we can then envision light-controlled piezo-transducers.

While electron-phonon coupling in ultrashort time scales has been investigated in depth in bulk solids in order to understand and control the emission of coherent phonons, artificial nanostructures have also opened a new possibility to tailor the spectrum of coherent acoustic phonons in particular. One prominent example of artificial nanostructures are semiconductors superlattices that are made of, usually, 1D growth of a sequence of nanometric semiconductor layers like $\mathrm{GaAs}$ and $\mathrm{Al}(\mathrm{Ga}) \mathrm{As}$. By choosing the the superlattice period with an accuracy down to a single atomic layer, we control the modefolding and hence the phononic band structure $[130,140]$. 
Superlattices can be used either for controlling the generation or to selectively enhance the detection at given frequencies (see Fig. 6b). In order to enhance the emission/detection of coherent acoustic phonons, quantum wells, and quantum dots have been employed to control the spectrum of acoustic phonon emission because these confined nanostructures are known to reveal enhanced electron-phonon coupling [141,142]. Another intriguing set of materials are electronically correlated solids, like correlated oxides, ferroelectrics or multiferroic compounds. These materials exhibit large electron-phonon coupling parameters, large electro-mechanical response and also augmented piezoelectric coefficients. Hence they open new pathways for exciting and controlling lattice and electron dynamics simultaneously [131,143-145]. Coherent phonon dynamics in topological insulators is also a subject of active research [146-149] where the electron-phonon coupling appears to set a limit on the natural spin polarized surface electrons transport [150,151]. Moreover, in magnetic materials, the interaction of acoustic phonons with spins opens the pathway for new functionalities. It has been demonstrated that picosecond strain pulses can lead to a coherent magnetization precession [152] or inverse magnetostriction [153]. Understanding electronphonon interaction in tailored quantum structures offers guidance to generate novel electrically driven acoustic phonon amplification processes. These observations served as an inspiration for devices similar to the lasers, based on sound amplification by stimulated emission (SASER). Pioneering work towards the SASER and active on-chip phonon devices has been performed by the group of Kent $[154,155]$. The concept of SASER is also currently tested with a different approach involving electromechanical oscillator [156] or opto-mechanical cavities [157], for which more details will be given in Section 4 .

All the previous examples are based on absorbing materials. The case of below band gap photoexcitation was hardly investigated in the last 20 years, mainly because of the low efficiency of the mechanism to generate coherent phonons through electrostriction $[158,159]$. A renewed interest appeared since the birth of femtosecond terahertz sources. With efficient terahertz source, with electric fields of the order of $100 \mathrm{kV} / \mathrm{cm}$ [160], it is now possible to envision a direct coupling of external field to phonons. The advantage of this excitation mechanism is to prevent, at the least, the lattice heating induced by hot electronrelaxation. It offers an exciting possibility as well to trigger phase transitions via the excitation of certain lattice modes, if coupling is achieved with soft modes that drive a displacive phase transition, or triggering the system to become superconductive [161].

\subsection{Probing nanostructures and phonon dynamics}

As discussed above, a broad spectrum of coherent acoustic phonons (gigahertz-terahertz) as well as optical phonons can be controlled through ultrafast laser pulses. Among the possible applications, testing the elasticity of nanostructures and interfaces has attracted a lot of attention, because of the crucial interest for materials science and nanotechnologies. For these investigations, coherent acoustic phonons are preferred to optical phonons because their group velocity is non-zero and they can propagate in nano- and microstructures. Due to this propagation ability, they can penetrate where light (pump or/and probe) sometimes can not reach. Moreover, with the high frequency range that can be reached, as small as $10 \mathrm{~nm}$ acoustic wavelength can be obtained, which enables high resolution optimal for testing nanostructures. Many properties of nanostructures have been studied by monitoring coherent acoustic phonons. Among them, the sound velocity is one of the most investigated physical properties in thin films and nanostructures [111,162-167]. Depth profiling of physical properties has been successfully achieved because of the possibility to monitor the propagation of coherent acoustic phonons in time and in space; for example, to evaluate the spatial extension of a $\mathrm{p}-\mathrm{n}$ semiconductor junction [168], to obtain evidence of some mechanical and optical inhomogeneities in nanoporous low- $\mathrm{k}$ thin film $[169,170]$, or to visualize the membrane and the nucleus in a single biological cell [171]. Probing the interfaces and the nano-contact (adhesion) has also become a challenge in nanometrology due to the fact that new functional materials are artificial materials assembled of different layers. It has been possible to evaluate the role of contacts between two solids (van der Waals, covalent bonds) on the transmission of coherent acoustic phonons at interfaces $[166,172-174]$ and on the sound velocity in arrays of nanoparticles $[175,176]$ (see Fig. 7) by measuring the time of flight of coherent acoustic phonons in various nanostructures.

A further approach pursued to gain better insight into phonon dynamics under confinement is the study of plasmonic excitations in metallic nanoparticles. The methodology has recently reached maturity to investigate not only ensembles but also single nanoparticles. An extensive review of the current progress in the study of coherent phonons in nanoparticles can be found in [177].

As reported above and discussed in Sections 2.2 and 2.3, engineering superlattices $[130,140]$ or suspended membranes $[60,178,179]$ allows one to tailor the acoustic phonons spectrum. Furthermore, it is possible to study the lifetime of specific vibrational modes in nanostructures. Therefore, this opens new perspectives in the study of phonon-phonon scattering processes with a narrow band spectrum spectroscopy. Contrary to broad band spectroscopic techniques, this approach allows one to evaluate directly the phonon lifetimes, which is crucial for understanding thermal conductivity (see Sect. 2). There are some intrinsic limitations of this technique, such as the relatively low frequency range $(<1 \mathrm{THz})$ compared to the usual full phonon spectrum of a solid. Among recent studies, one can note the evaluation of lifetimes of phonons in acoustic nanocavities [180] and in Si membranes [60,178]. The lifetime is extracted from the damping of the laserinduced mechanical resonance in the membranes. Figure 8 a displays several eigenmodes of a self-supported membrane that can be generated and detected. The direct damping of each of the modes provides a way to evaluate 

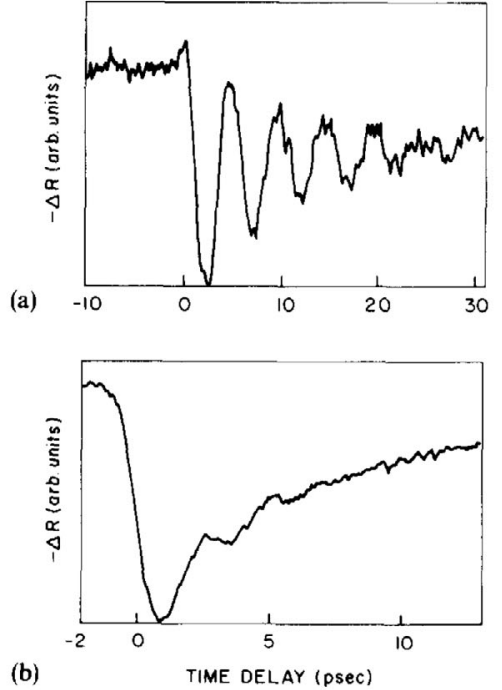

(a)

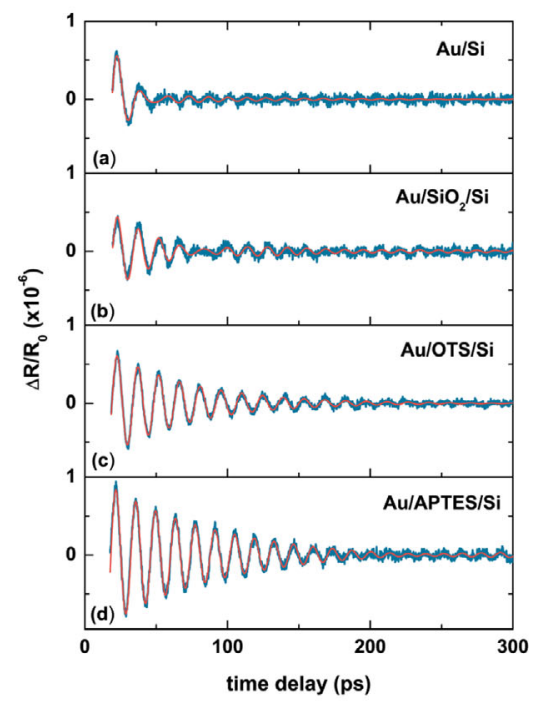

(b)

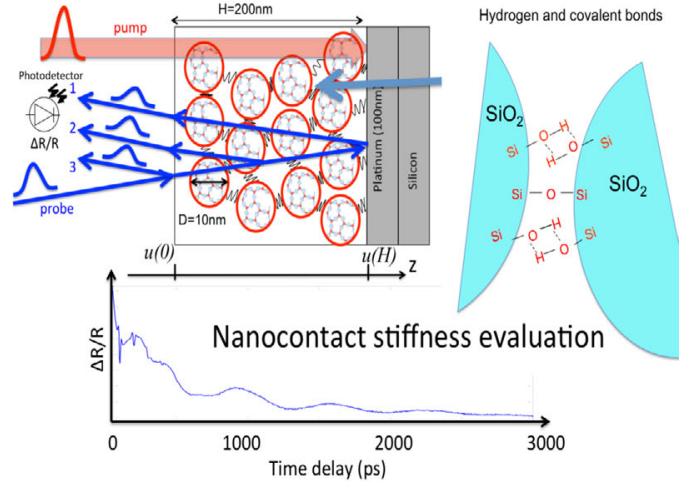

(c)

Fig. 7. (a) Light-induced mechanical oscillation of a metallic thin film deposited on a substrate with a poor (top) and good (bottom) adhesion. A poor adhesion prevents the mechanical energy to be transmitted into the substrate and favors a long oscillation lifetime [172]. (b) Influence of the molecular layer at the interface between the gold film and Si substrate onto the coherent acoustic phonons transmission (i.e. lifetime of phonons in gold film) [173]. (c) Light-induced mechanical resonance of colloidal films, the cohesion of which is determined by either van der Waals, hydrogen or covalent bonds. The analysis of the resonance frequency allows to evaluate the speed of sound along their chains in colloidal films and hence the nanocontact stiffness between nanoparticles [175].

(a)

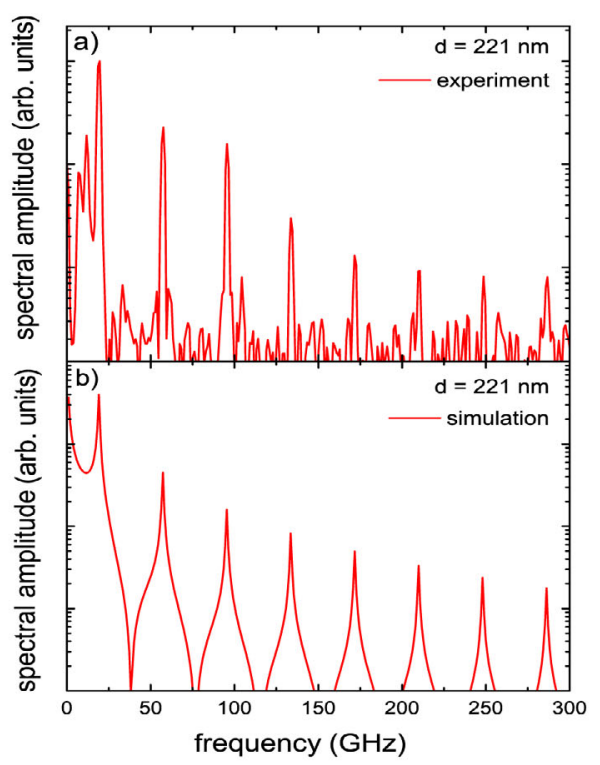

(b)

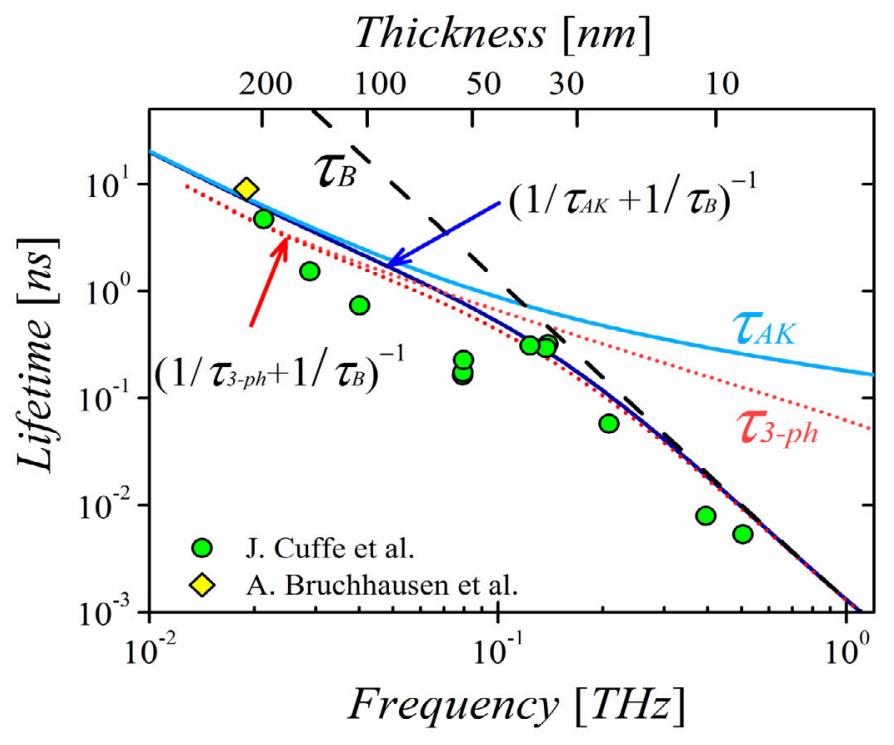

Fig. 8. (a) (top) Acoustic spectrum of laser-induced mechanical resonance of a thin silicon membrane where several harmonics have been generated and detected within a single membrane [178]. (bottom) Calculated eigenmodes frequencies. (b) Phonon lifetime of the first-order dilatational mode in free-standing silicon membranes as a function of frequency [60]. The black dashed line shows the contribution to the finite phonon lifetime from boundary scattering and the solid cyan and dotted magenta lines show the contributions of the modified Akhieser, $\tau_{A k}$, and Landau-Rumer, $\tau_{3-p h}$, models, respectively. The total phonon lifetimes are calculated using Matthiessen's rule. The solid blue and dotted red lines are the predictions from modified-Akhieser and Landau-Rumer models, respectively. 
the phonon lifetimes. As shown in Figure 8b, the lifetime decreases dramatically with frequency, as expected. The analysis of the frequency dependence of phonon lifetimes provides an opportunity to test the different theoretical models predicting phonon attenuation, highlighting the role of intrinsic phonon scattering or extrinsic surface roughness scattering. Intrinsic phonon lifetimes are modeled employing two different approaches, commonly referred to as Landau-Rumer model [181] and modified Akhieser model [182], which take into account the modification of the thermal conductivity of the membrane [183]. The surface scattering is modeled with the Ziman approach, incorporating a wavelength-dependent specularity parameter [184]. The validity of each model depends on the wavelength of the absorbed phonon, $\lambda$, and the mean free path of the thermal phonons, $\Lambda_{m f p}$. If the $\lambda$ of the acoustic wave is much longer than the typical $\Lambda_{m f p}$ of the thermal phonons, i.e., $\mathrm{f} \tau_{T h} \ll 1$, where $\tau_{T h}$ is the lifetime of the thermal phonons, we can assume that the acoustic wave interacts with the whole spectrum of thermal phonons. This range is known as the Akhieser regime. But if $\lambda$ is much less than $\Lambda_{m f p}$, i.e., $\mathrm{f} \tau_{T h} \gg 1$, then the phonon attenuation can be better described by the Landau-Rumer formalism and the phonon attenuation is due to threephonon interaction processes. By taking into consideration these different scattering processes, it was found that the combination of these empirical models, properly parameterized, describes the trend of phonon lifetimes quite well over several orders of magnitude, varying from being dominated by intrinsic effects for thicker membranes, to being limited by surface roughness for thinner membranes.

\section{Nano- and optomechanics}

The discrete vibrational modes of freely suspended nanoor microstructures are explored in the context of nano- or cavity optomechanical systems. The ongoing rise of this field is fueled by a broad range of possible applications: On the one hand, the narrow resonance linewidths and small masses of nanomechanical resonators make them susceptible to minute changes in their environment such that narrow-band filters and responsive sensing devices can be envisaged. On the other hand, their ability to couple to various degrees of freedom such as light, electronic transport or magnetic properties makes them a versatile linking element in hybrid nanosystems. While the applications mentioned previously can be beneficial for practical devices operating in the classical physics realm, a nanomechanical resonator cooled to a low enough temperature will enter the quantum mechanical regime [185] where novel insights into fundamental questions of physics or stimuli for quantum technologies can be expected [186,187].

\subsection{Nanomechanics}

Nanomechanical systems [188] are typically realized using top-down nanofabrication, but also integrate bottomup elements such as carbon nanotubes, graphene or other atomically thin membranes [189]. Their vibrational

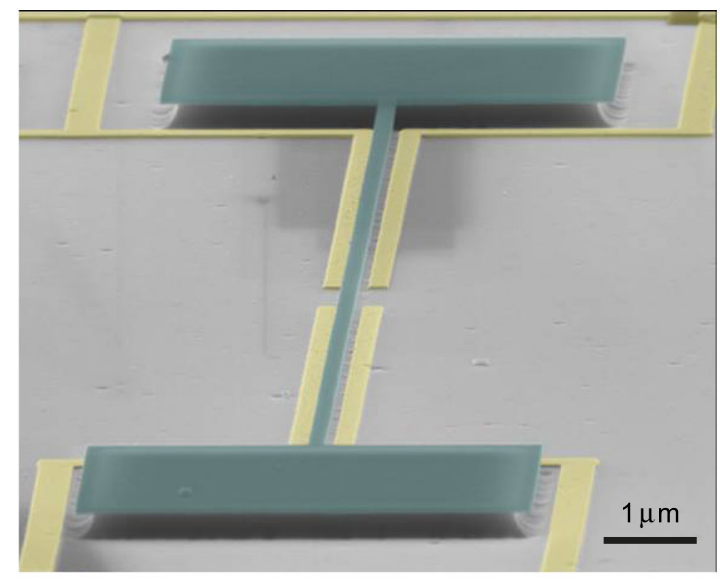

Fig. 9. Nanomechanical resonator. The depicted example is a $35 \mu \mathrm{m}$ long SiN string with a cross-section of $100 \mathrm{~nm} \times 200 \mathrm{~nm}$ (adapted from [196]). Substrate-borne electrodes are for dielectric transduction.

properties are conveniently described by elasticity theory $[188,190]$. For example, the flexural modes of a doubly clamped nano-string, such as the one depicted in Figure 9, are modeled by Euler-Bernoulli beam theory. In the linear response regime, they can be expressed as a harmonic os-

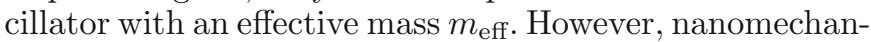
ical vibrations can also be thought of as standing acoustic waves and hence as discretized, low frequency acoustic phonon modes. This framework is particularly intuitive in the quantum regime of nanomechanics, where only few phonons populate the mode. Without cavity-assisted cooling techniques (which will be described in the following subsection) the quantum ground state of a mechanical resonator is challenging to reach as a consequence of the condition $\hbar \Omega_{M} \gg k_{B} T$, and has only been reported for a $6 \mathrm{GHz}$ bulk acoustic resonator mode cooled to millikelvin temperatures in a dilution refrigerator [185]. For a typical nanomechanical resonator with an eigenfrequency of several megahertz up to a few gigahertz, room temperature vibration implies an extremely large occupation of the phononic mode, hence ensuring operation in the classical regime.

Nanomechanical resonators are frequently controlled by electrical means. In many ways, the resulting nanoelectromechanical systems (NEMS) are scaled-down versions of the well-established MEMS, as discussed in [191]. A range of review articles details the transduction of nanomechanical resonators by electrical or by optical means [192,193], their dissipation [194] as well as their nonlinear dynamics [195], and can be consulted for further information.

Recent years have seen a quest for large mechanical quality factors which can e.g. be realized in strongly pre-stressed resonators [197-199], novel materials such as crystalline diamond [200] or strained crystalline materials [201], or by suppressing clamping losses with suitable architectures [202-204]. The realization of large mechanical quality factors allows to address questions including the nonadiabatic dynamics of strongly coupled modes [205], the coherent control of the mechanical 


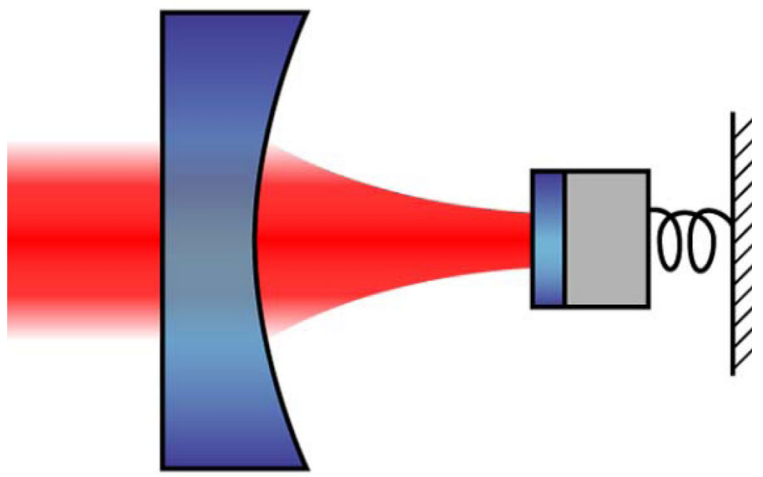

Fig. 10. Schematic of a cavity optomechanical system (from Wikipedia).

state $[206,207]$ as well as a more thorough understanding of nanomechanical coherence [206,208,209].

Progress in the control of nanomechanical systems has enabled to harness the coupling of mechanical resonators [210] or harmonic modes [211-213]. This allows to target the nonlinear dynamics $[214,215]$ beyond a single resonator mode [216], which can be employed to realize e.g. electromechanical phonon lasing [157] or phase synchronization [217].

The technology of NEMS has vast potential for sensing applications, not only for their ultra-high sensitivity enabling these devices to resolve a single electron spin or only a few nuclear spins $[218,219]$, forces down to the zeptonewton regime [220] or masses of only a few yoctograms [221], but also for mass-market applications such as gyroscopes or other types of accelerometers [222]. Furthermore, NEMS can be engineered to be CMOS compatible such that full integration of sensors, switches or narrow-band filters into CMOS architectures can be envisioned. Another area of interest is bioNEMS, which has a vast potential for biosensing applications [223,224].

Furthermore, nanomechanical systems feature the remarkable property to couple not only to acoustical excitations but also to single electrons [225-227] and spins [228-230], which makes them an interesting candidate to realize and to control hybrid nanosystems. In this context also the optomechanical coupling to photons should be mentioned, which will be detailed in the following subsection.

\subsection{Cavity optomechanics}

Cavity optomechanics explores the parametric coupling of a mechanical resonator to an optical cavity mode (Fig. 10). Hence optomechanical coupling relies on the modification of the optical properties of the cavity by a displacement of the mechanical resonator $x$. Various review publications have covered the developing state of the art over the past years [5,231-233], such that the following discussion is only intended to provide a coarse overview. In particular, the interested reader is referred to the recent review
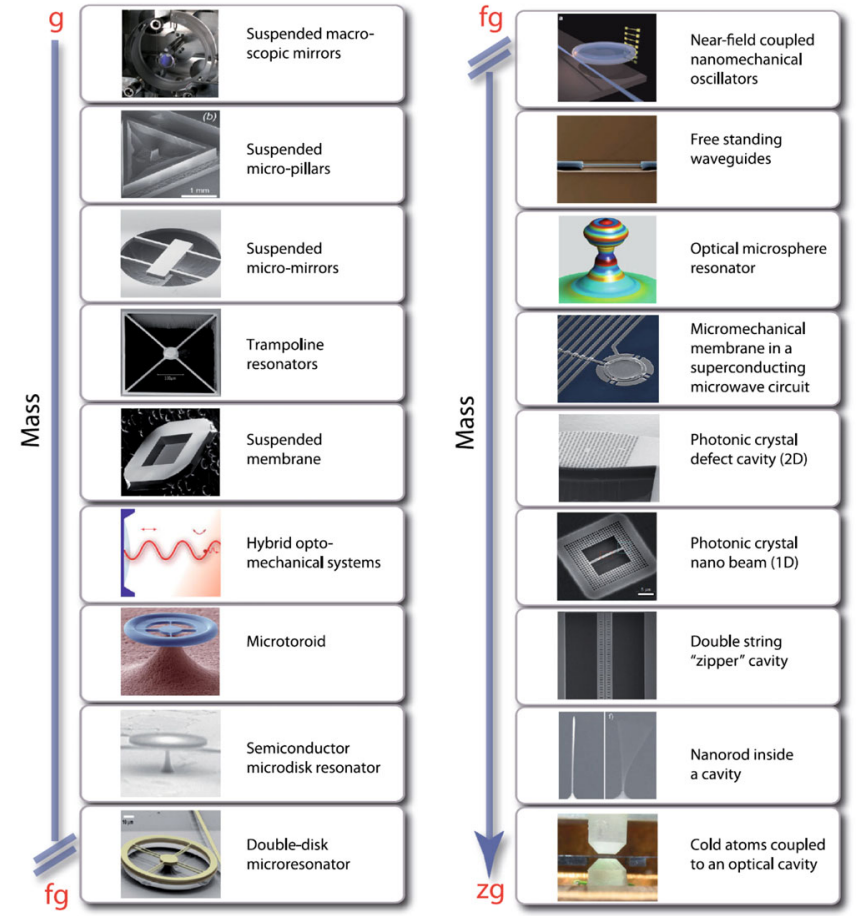

Fig. 11. Realizations of cavity optomechanical systems (Fig. 7 from [5]). Copyright (2014) by The American Physical Society.

by Aspelmeyer et al. [5] or to the Springer textbook edited by the same authors [234] for more details.

A landmark cavity optomechanical system is a Fabry-Pérot cavity with a moveable mirror, where the resonator displacement directly translates into a change in cavity length and thus eigenfrequency. This is an example for dispersive optomechanical coupling which is defined by a displacement-dependent cavity eigenfrequency $\omega_{\text {cav }}(x)$. In contrast, dissipative coupling is observed for a displacement-dependent optical linewidth $\kappa(x)$. Realizations of cavity optomechanical systems are diverse, including nanostructures as well as kilometerscale gravitational wave detectors, hybrid as well as integrated devices, optical as well as microwave cavities, and mechanical resonators covering zeptogram to kilogram masses (Fig. 11). One implementation which is particularly close to the field of phononics is based on onedimensional photonic-phononic crystals, so-called optomechanical crystals [235-237], which are also referred to as phoXonic crystals [238]. Recently, optomechanical crystals with a complete bandgap have been demonstrated [239]. Particularly interesting are two-dimensional implementations [240] which promise to enable optomechanical arrays [241] with interesting topological properties [242]. A novel class of optomechanical systems are levitated nanoparticles which promise to greatly enhance the coherence time [243-246]. Furthermore, it should be noted that the framework of cavity optomechanics can equally be applied to the microwave realm, where a (superconducting) microwave circuit replaces the optical cavity [247]. These so-called cavity electromechanical (or circuit electromechanical) systems offer the benefit of relatively large 
optomechanical coupling [248], and allow for the integration of further circuit components, such as an artificial two-level system [249], or for bottom-up mechanical resonators such as graphene sheets [250].

Despite their variety all optomechanical systems (OMS) share the simple concept that a mechanical mode alters an optical mode. The resulting interdependence between mechanical motion and optical properties effectively acts as an intrinsic feedback loop that generates optomechanical backaction. The underlying interaction between mechanical motion and optical properties that mediates optomechanical coupling is caused by lightinduced forces such as the radiation pressure force or photothermal forces. These forces are typically retarded due to a finite cavity lifetime or thermal conductance, respectively, which gives rise to dynamical backaction.

For many optomechanics scenarios it is sufficient to linearize the interaction $\omega_{\text {cav }}(x) \approx \omega_{\text {cav }}-G x+\ldots$, defining the frequency pull parameter $G=-\frac{\partial \omega_{\text {cav }}}{\partial x}$ which indicates the induced cavity eigenfrequency shift per resonator displacement of a dispersive optomechanical system. The frequency pull parameter plays an important role to quantify the optomechanical coupling strength which is conveniently expressed as a frequency $g=\sqrt{n_{\text {cav }}} G x_{\text {zpf }}=$ $\sqrt{n_{\text {cav }}} g_{0}$, where $n_{\text {cav }}$ is the number of photons circulating in the cavity and $x_{\mathrm{zpf}}=\sqrt{\frac{\hbar}{2 m_{\mathrm{eff}} \Omega_{M}}}$ is the amplitude of the mechanical zero-point fluctuations of the resonator. While the optomechanical coupling $g$ depends on the laser intensity pumping the cavity, a more fundamental parameter to characterize the interplay between phonons and photons is the single-photon optomechanical coupling strength $g_{0}=G x_{\mathrm{zp} f}$ which quantifies the interaction between a single photon and a single phonon.

Linear optomechanics describes many of the features observed in optomechanical systems, including the optical spring effect as well as the optomechanically induced damping which can be either positive or negative, depending on the detuning $\Delta=\omega_{\mathrm{L}}-\omega_{\text {cav }}$ of the laser frequency $\omega_{L}$ with respect to the optical cavity eigenfrequency. For a blue detuned laser, i.e. $\Delta>0$, the additional backaction damping $\Gamma_{\text {cav }}$ is negative. Thus the total damping is diminished upon increasing the laser power and will eventually become negative. This denotes the onset of instability and self-induced mechanical oscillation. The regime of optomechanical parametric instability features a rich nonlinear dynamics and is reminiscent of a laser above threshold [251,252]. For a red detuned laser with $\Delta<0$ the backaction damping $\Gamma_{\text {cav }}$ is positive, such that the total damping is increased upon increasing the laser power. This gives rise to optomechanical cooling [253-257]. Assuming radiation pressure backaction, the minimum attainable temperature is ultimately limited by the fluctuations of the radiation pressure force due to photon shot noise, such that ground state cooling is enabled for various scenarios. Typically dispersive optomechanical coupling in the resolved sideband regime $\left(\Omega_{M}>\kappa\right)$ is considered [258,259], but also other scenarios including dissipative coupling or even photothermal backaction are discussed. The prospect of entering the quantum regime of nanomechanics has driven the field of cavity optomechanics over the past decade, and after the first groups reported backaction cooling to the quantum ground state in 2011 using an optomechanical crystal [236,237] and a cavity electromechanical system operating in the microwave regime [260], respectively, the field of quantum optomechanics is rising [261-265].

Another important figure of merit is the so-called cooperativity $C=\frac{4 g^{2}}{\kappa \gamma_{M}}$, a dimensionless number which relates the optomechanical coupling $g$ to the optical and mechanical loss rates $\kappa$ and $\gamma_{M}$, respectively, and which is e.g. employed to quantify the strength of optomechanically induced transparency [266]. As soon as the cooperativity exceeds the number of thermally excited resonator phonons, $C>n_{\text {th }}$, the system enters the quantum coherent regime, in which quantum states can be swapped between the photonic and the phononic subsystem before the system decoheres and which hence enables the realization of mechanical quantum states.

Beyond linear optomechanics, systems can be engineered to exhibit a higher order dispersive optomechanical coupling, such as e.g. $x^{2}$-coupling in which the cavity eigenfrequency is linked to the square of the mechanical displacement $[267,268]$. In the quantum regime, $x^{2}$-coupling enables the quantum non-demolition (QND) measurement of the phonon number, and hence represents an major goal for quantum optomechanics. Furthermore, the linearized description of optomechanics fails for very large coupling strengths: In the strong coupling limit $g>\kappa$, the driven optical and the mechanical mode hybridize [269]. An even tighter requirement is set by the single-photon strong coupling regime, in which the interaction between single photons and photons becomes coherent. This condition translates into $g_{0}>\kappa, \gamma_{M}$, and corresponds to the situation $C_{0}=\frac{4 g_{0}^{2}}{\kappa \gamma_{M}}>1$. The single-photon strong coupling regime enables the creation of non-Gaussian quantum states of the mechanical resonator [270]. To date, this important milestone has not been reached, despite the fact that promising optomechanical systems have been identified, including e.g. [271,272].

The exploration of non-classical phenomena allows to target unresolved questions in fundamental physics such as the entanglement of macroscopic objects or tests of quantum theories involving gravity [273]. Furthermore, optomechanical systems represent a versatile platform for the development of ultrasensitive, potentially quantum limited sensing elements [274,275]. At the same time, they can be envisioned to act as building blocks for quantum information technology, and used for long time storage of quantum states or as transducers between optical photons used in (quantum) communication and microwave photons easily compatible with chip-based data processing [276-278].

\section{Perspective and applications}

Phonons play a crucial role in the performance of electronic devices, limit the efficiency of thermoelectric 
materials, proposed to be the key for the efficiency of various processes in biology and provide possibilities for new applications in data processing, health, safety and security.

Heat dissipation sets the limits for the performance of most of today's electronic applications. For example, the operation speed of integrated circuits and the efficiency of LED lighting are limited by the efficiency of heat removal from the active parts of devices and through the interfaces coupling the devices to the surroundings. Packaging techniques and development of better thermal interface materials (TIM) is a good example of applications in which understanding and controlling phonon propagation can generate substantial improvements in thermal conductance and heat removal and, consequently, lead to improved device performance and lower power consumption. Also, with shrinking dimension and active volume of the devices reducing down to a few tens of $\mathrm{nm}$ and even below, i.e., less than the average mean free path of heat carrying acoustic phonons, understanding phonon scattering and the consequences to heat dissipation presents new challenges to design device and micro- and nanoelectronic system integration.

Surface and bulk acoustic wave (SAW, BAW) devices represent applications exploiting long wavelength lattice vibrations - "sound" - in microelectronics $[279,280]$. The operation is based on the coupling between electric fields and lattice vibrations in piezoelectric materials. The devices enable RF-MEMS filter technology for mobile phones and other hand-held applications, operating typically in the $1-10 \mathrm{GHz}$ range depending on the dimensions. A different and interesting application based on surface acoustic waves is electron pumping. In SAW devices the strain field creates traveling potential wells for electrons and holes, and the wells can be used to localise and transport the carriers [281]. The carriers are usually photoexcited and are trapped by the strain field propagating in a controlled direction. Multiplexing the electronic signal on chip can be achieved by employing different surface acoustic waves $[7,282]$. Phonon-induced strain fields can also manipulate barrier heights in junctions and this effect has been demonstrated with Schottky barriers [283]. The challenge to create deep enough moving potential wells by strain for practical devices remains to be solved.

Optomechanics, described in detail in the previous section, takes the advantage of coupling phonons and photons with phonons representing the mechanical degree of freedom in the system. This makes it possible, for example, to modulate telecommunication wavelengths at $\mathrm{GHz}$ range on very compact single chips at room temperature [239] and store quantum information at low temperatures.

In addition to storing, quantum information can also be transferred using propagating phonons instead of photons, i.e., to replace light with sound [284]. Spin-mediated energy transport, or magnons, in spin-chain materials is an emerging research field. Relatively high and strongly crystallographic direction dependent thermal conductivities have been measured in antiferromagnetic bulk com- pounds [285]. Spin-dependent Seebeck and Peltier effects can potentially be exploited in energy harvesting and in coolers, and spin caloritronics in nanoscale heat engines [286].

Terahertz spectroscopy has a great promise in the field of medical imaging and for safety measures. This method can reveal skin cancer and other tumors at early stages and give images of structures which are optically opaque, in vivo [287] and in vitro [288]. In addition to imaging, $\mathrm{THz}$ radiation can couple to the vibrational modes of molecules and can provide information regarding biological bonds. The four nucleobases of DNA have different vibrational spectra and can be differentiated [287]. Terahertz technology has also been used as screening technology at the airports to prevent people from entering vehicles with concealed weapons, including non-metal edged weapons. The vibrational frequencies of the chemical bonds allow the recognition of explosives [289]. This technology also provides tools for metrology and standards. Although hampered currently by the lack of stable sources and detectors, the number of companies producing instruments for power measurement and time and frequency domain spectroscopy is steadily increasing [290].

However, terahertz spectroscopy falls short regarding spatial resolution. The resolution of terahertz imaging is in the range of 100 's of $\mu \mathrm{m}$, limited by the electromagnetic wavelength used, while in several applications much higher resolution is needed. Ultra-fast acoustics provides an alternative to achieve sub-micron resolution. In this technique, ultra-fast optical pulses generating thermoelastic excitations in a thin metal film are used to provide picosecond acoustic phonons which can be used for non-invasive imaging with potentially sub-100 nm resolution [291]. The phonons scatter from inclusions and interfaces in the matrix and this makes it possible to devise a technique for 3-dimensional non-destructive imaging of objects. The 3D imaging, however, requires new type of algorithms and heavy computation, making the approach challenging [292]. This type of nanoscale ultrasonics can be used to inspect microscale cracks and inhomogeneous spots, e.g., in vessels used in chemical industry and in nuclear plants. Non-invasive depth-profiling of acoustical, optical and acousto-optical parameters of optically transparent inhomogeneous materials with sub-100 nm resolution has been already achieved through the application of time-domain Brillouin scattering, and cold picosecond ultrasonic interferometry $[169,170,293]$. The perspective here is to reach sub-10 nm in depth resolution and to improve lateral resolution, which is currently limited by laser focusing.

Quantum biology is an emerging field with the ambition to reveal the mechanisms of processes in biological systems, e.g., photosynthesis, and to explain the origin of their surprisingly high efficiency [294]. The key reason seems to be the coupling of the specific excited system to its environment and the benefit from the coherent vibrations of the surrounding molecules. When an excitation event brings the system to a non-equilibrium state, the back-action by the environment leads to a long lasting 
coherence or even a quantum coherence in the system and helps to guide the energy efficiently to the desired location, in case of photosynthesis, to the reaction center converting light to chemical energy [295]. The coupling between excitons and the vibrations, showing oscillation bands in the range of a few hundreds of $\mathrm{cm}^{-1}$, can explain the high efficiency of the solar energy conversion of plants by ultra-fast charge separation via coherence of charge transfer states and excitons in the specialized pigmentprotein complexes dedicated to light trapping, in spite of the noise in the surroundings [296]. Understanding these mechanisms may open routes in long term to develop new types of solar cells with almost hundred percent efficiency, providing a potential solution to the ever increasing need of energy.

Thermoelectrics represents a field that has largely benefitted from the recent advances in phononics. Thermoelectric devices can be used as power generators for energy harvesting or as Peltier cooling elements [297]. The performance of thermoelectric devices depends on the dimensionless figure of merit of the material, $Z T=\frac{\sigma S^{2}}{\kappa_{p h}+\kappa_{e l}} T$, in which $T$ is the average temperature between the cold and the hot ends of the device, $\sigma$ is electrical conductivity, $S$ is the Seebeck coefficient of the material and the two $\kappa$ represent the phononic and the electronic thermal conductivities. The limiting factor to reach high figure of merit for bulk materials typically is the phononic thermal conductivity. Only after the introduction of nanostructured materials in the late 90's the ZT has been improved above one at room temperature [298]. The progress is based on blocking the propagation of phonons by nano and microstructuring of the material. The mean free path of heat carrying acoustic phonons span from nanometre to several micrometres and by building the materials in an appropriate way, the thermal conductivity can be largely reduced. Nanocrystalline bulk materials have shown $Z T$ values above one [299], and hierarchically constructed materials designed to scatter the phonons with all the wavelengths can yield even higher values [300]. The above mentioned materials are typically rather complex and contain toxic elements or materials with low abundance. The recent advances in reducing the thermal conductivity in silicon based materials shows that the dominating microelectronics materials also have a promise as thermoelectric devices [70]. The reduction of thermal conductivity by a factor of 20 in thin silicon membranes while maintaining a good electrical conductivity and Seebeck coefficient suggests that a relatively high figure of merit can be achieved in pure silicon.

Probing the behavior of phonons experimentally is not straightforward and calls for new techniques and new dedicated tools. Two-laser Raman thermometry is a good example of a new approach that facilitates the determination of thermal conductivity of free-standing silicon membranes [301]. The investigation of phonon propagation and lifetimes requires high temporal and spatial resolution and represents an emerging market for new equipment. Novel tools, such as asynchronous optical sampling (ASOPS), can give detailed information about the life- times and scattering mechanisms [70,302] and will enhance the understanding of the role of phonons in materials, nanostructures and devices. Altogether, understanding and controlling the behavior of phonons will help to improve the properties and performance of a wide variety of current electronic devices through heat management and to create new applications by exploiting the interactions between phonons, between phonons and photons and phonons and electrons.

\section{Conclusions}

In this colloquium paper we have summarized the recent achievements, perspectives and open challenges of the thriving field of nanophononics. Major progress has been recently made both in theory and experiments in several fundamental phenomena related to the understanding and design of phonon properties at the nanoscale.

At the theoretical level early empirical models, e.g. Callaway and Holland models [184], based on a simplified solution of the Boltzmann transport equation, have been overcome by the development of parameter-free atomistic methods to calculate the effect of localization and dimensionality reduction on heat transport [27-29]. Firstprinciples lattice dynamics methods are complemented by MD and GF approaches, which extend the applicablity of theory to complex systems [70] and a broader variety of phenomena, including near-field radiation [88]. Theoretical predictions can now be verified experimentally to unprecedented levels of detail: Brillouin light scattering enables the direct measurement of phonon dispersion relations in nanoscale materials for acoustic phonons up to hundreds of gigaherz in frequency [60]. Thermal conductivity spectroscopy enables the measurements of heat carriers mean free paths, although the application of this approach has been so far limited to silicon $[51,55]$. Moreover, advances in synchrotron radiation-based dynamic studies are relating spatially resolved simultaneously structural and lifetime analysis of modal distributions and continue to be developed. Such deeper understanding of phonon transport has fostered the development of new nanomaterials and devices with engineered phononic and thermal properties, ranging from hierarchically nanostructured high-performance thermoelectric materials [4], to phononic crystals with engineered phononic bandgaps, and metamaterials that allow one to control thermal fluxes as in switches, rectifiers or cloaks $[72,73]$.

Progress in understanding the interaction of light with the lattice dynamics of materials has favored the development of coherent acoustic phonon sources (SASER) [154] and advances in fast terahertz spectroscopic techniques and imaging, to probe nanostructures [176], composite materials and interfaces [169], and even biological systems [171]. Photons and phonons couple in optomechanical cavities and resonators, which provide access to quantum phenomena [5], paving the way to quantum state variables. Improvement of materials and of nanofabrication techniques led to the development of high-quality and 
highly efficient nanomechanical resonators to be exploited as sensors, switches and narrow-band filters.

So far the highlighted achievements were reached by disjoint efforts of separate communities. However, the accomplishments so far are in a very early phase and of limited applicability, regarding the thermodynamic conditions of operation, the type of materials and the kind of phenomena that can be probed. Further progress in controlling phonons is highly needed (i) to achieve more efficient cooling - either active or passive - from hot spots in nanoelectronic devices, (ii) to envisage new state variables for information and communication, (iii) to improve the quality factor of nanomechanical resonators and sensors, (iv) to develop new coherent phonon sources for acoustic and terahertz spectroscopy, (v) to improve thermoelectric energy conversion, and, (vi) to improve imagining and diagnostics in medical therapy. Hence, it is essential to establish phononics as a priority transversal research topic, and to address the broad nanophononics community into a collaborative effort leading to rapid progress toward the objectives illustrated in this colloquium, with beneficial consequences at the level of both basic science and technology.

\section{Author contribution statement}

Sebastian Volz, Jouni Ahopelto, Pascal Ruello, Eva M. Weig, Thomas Dekorsy, Clivia M. Sotomayor-Torres and Davide Donadio contributed equally to this work.

The authors acknowledge financial support by the European Commission FP7 FET Open project EUPHONON, grant agreement No. 612086. The views expressed here are the sole responsibility of the authors.

\section{References}

1. E. Pop, Nano Res. 3, 147 (2010)

2. Z. Yan, G. Liu, J.M. Khan, A.A. Balandin, Nat. Commun. 3, 827 (2012)

3. G.J. Snyder, E.S. Toberer, Nat. Mater. 7, 105 (2008)

4. K. Biswas et al., Nature 489, 414 (2012)

5. M. Aspelmeyer, T.J. Kippenberg, F. Marquardt, Rev. Mod. Phys. 86, 1391 (2014)

6. R.P.G. McNeil et al., Nature 477, 439 (2011)

7. S. Hermelin et al., Nature 477, 435 (2011)

8. C. Rossignol et al., Appl. Phys. Lett. 93, 123901 (2008)

9. N. Zen et al., Nat. Commun. 5, 1 (2014)

10. J.-K. Yu et al., Nat. Nanotechnol. 5, 718 (2010)

11. N. Li et al., Rev. Mod. Phys. 84, 1045 (2012)

12. E.W. Montroll, J. Chem. Phys. 18, 183 (1950)

13. H. Baltes, E. Hilf, Solid State Commun. 12, 369 (1973)

14. D.G. Cahill et al., J. Appl. Phys. 93, 793 (2003)

15. A.I. Hochbaum et al., Nature 451, 163 (2008)

16. R. Chen et al., Phys. Rev. Lett. 101, 105501 (2008)

17. D. Li et al., Appl. Phys. Lett. 83, 2934 (2003)

18. M.P. Zaitlin, L.M. Scherr, A.C. Anderson, Phys. Rev. B 12, 4487 (1975)
19. M. Kazan et al., J. Appl. Phys. 107, 083503 (2010)

20. S. Volz, in Thermal Nanosystems and Nanomaterials, Topics in Appl. Phys. (Springer, Berlin, 2009), Vol. 118

21. S. Volz, Micro and Nano Heat Transfer, Topics in Appl. Phys. (Springer, Berlin, 2008), Vol. 107

22. M.N. Luckyanova et al., Science 338, 936 (2012)

23. J. Ravichandran et al., Nat. Mater. 13, 168 (2014)

24. I. Savic, D. Donadio, F. Gygi, G. Galli, Appl. Phys. Lett. 102, 073113 (2013)

25. S. Baroni, S. de Gironcoli, A. Dal Corso, P. Giannozzi, Rev. Mod. Phys. 73, 515 (2001)

26. A. Ward, D.A. Broido, D.A. Stewart, G. Deinzer, Phys. Rev. B 80, 125203 (2009)

27. G. Fugallo, M. Lazzeri, L. Paulatto, F. Mauri, Phys. Rev. B 88, 045430 (2013)

28. D.A. Broido et al., Appl. Phys. Lett. 91, 231922 (2007)

29. A. Cepellotti et al., Nat. Commun. 6, 1 (2015)

30. S. Lee, D. Broido, K. Esfarjani, G. Chen, Nat. Commun. 6, $7290(2015)$

31. A.J.C. Ladd, B. Moran, W.G. Hoover, Phys. Rev. B 34, 5058 (1986)

32. S. Volz et al., Phys. Rev. B 54, 340 (1996)

33. J.E. Turney, A.J.H. McGaughey, C.H. Amon, Phys. Rev. B 79, 224305 (2009)

34. O.N. Bedoya-Martinez, J.L. Barrat, D. Rodney, Phys. Rev. B 89, 014303 (2014)

35. R. Landauer, Phil. Mag. 21, 863 (1970)

36. N. Mingo, L. Yang, Phys. Rev. B 68, 245406 (2003)

37. W. Zhang, N. Mingo, T.S. Fisher, J. Heat Transfer 129, $483(2006)$

38. N. Mingo, Phys. Rev. B 74, 125402 (2006)

39. J.-S. Wang, J. Wang, N. Zeng, Phys. Rev. B 74, 033408 (2006)

40. S. Xiong, J. Ma, S. Volz, T. Dumitrica, Small 10, 1756 (2014)

41. L.V. Keldysh, Soviet Phys. J. Exp. Theor. Phys. 20, 1307 (1965)

42. D.A. Young, H.J. Maris, Phys. Rev. B 40, 3685 (1989)

43. I. Duchemin, D. Donadio, Phys. Rev. B 84, 115423 (2011)

44. P. Reddy, K. Castelino, A. Majumdar, Appl. Phys. Lett. 87, 211908 (2005)

45. C. Blanc et al., Appl. Phys. Lett. 103, 043109 (2013)

46. D.G. Cahill, Rev. Sci. Instrum. 61, 802 (1990)

47. Y.K. Koh, D.G. Cahill, Phys. Rev. B 76, 075207 (2007)

48. S. Lefèvre, S. Volz, Rev. Sci. Instrum. 76, 033701 (2005)

49. A. Sikora et al., Rev. Sci. Instrum. 83, 054902 (2012)

50. A. Sikora et al., Rev. Sci. Instrum. 84, 029901 (2013)

51. K.T. Regner et al., Nat. Commun. 4, 1640 (2013)

52. J.A. Johnson et al., Phys. Rev. Lett. 110, 025901 (2013)

53. E. Chávez-Ángel et al., Appl. Phys. Lett. Mater. 2, 012113 (2014)

54. J. Reparaz et al., Rev. Sci. Instrum. 85, 034901 (2014)

55. A.J. Minnich et al., Phys. Rev. Lett. 107, 095901 (2011)

56. L. Romain, Ph.D. thesis, Institut des Nanosciences de Paris, 2014

57. M. Maldovan, Nature 503, 209 (2013)

58. H. Lamb, Proc. R. Soc. London A 93, 114 (1917)

59. S. Bramhavar et al., Phys. Rev. B 83, 014106 (2011)

60. J. Cuffe et al., Nano Lett. 12, 3569 (2012)

61. B. Graczykowski et al., New J. Phys. 16, 073024 (2014)

62. A. Shchepetov et al., Appl. Phys. Lett. 102, 192108 (2013) 
63. G. Gopalakrishnan et al., Phys. Rev. Lett. 110, 205503 (2013)

64. L. Maerten, A. Bojahr, M. Bargheer, Ultrasonics 56, 148 (2015)

65. Z. Liu et al., Science 289, 1734 (2000)

66. B. Graczykowski et al., Phys. Rev B 91, 075414 (2015)

67. B.L. Davis and M.I. Hussein, Phys. Rev. Lett. 112, 055505 (2014)

68. Y. He et al., ACS Nano 5, 1839 (2011)

69. I. Maasilta and A.J. Minnich, Phys. Today 67, 27 (2014)

70. S. Neogi et al., ACS Nano 9, 3820 (2015)

71. M.S. Dresselhaus et al., Adv. Mater. 19, 1043 (2007)

72. H. Xu et al., Phys. Rev. Lett. 112, 054301 (2014)

73. T. Han et al., Phys. Rev. Lett. 112, 054302 (2014)

74. M.R. Wagner et al., arXiv:1511.07398 (2015)

75. D. Schneider et al., Nano Lett. 12, $3101(2012)$

76. E. Alonso-Redondo et al., Nat. Commun. 6, 8309 (2015)

77. V.M. García-Chocano, J. Christensen, J. SánchezDehesa, Phys. Rev. Lett. 112, 144301 (2014)

78. M.S. Kushwaha, P. Halevi, L. Dobrzynski, B. DjafariRouhani, Phys. Rev. Lett. 71, 2022 (1993)

79. P.A. Deymier, Acoustic Metamaterials and Phononic Crystals (Springer, Berlin, 2013)

80. D. Polder, M. Van Hove, Phys. Rev. B 4, 3303 (1971)

81. K. Joulain et al., Surf. Sci. Rep. 57, 59 (2005)

82. A. Narayanaswamy, S. Shen, G. Chen, Phys. Rev. B 78, 115303 (2008)

83. S. Shen, A. Narayanaswamy, G. Chen, Nano Lett. 9, 2909 (2009)

84. E. Rousseau et al., Nat. Photon. 3, 514 (2009)

85. M. Francoeur, Nature Nano 10, 206 (2015)

86. M. Prunnila, J. Meltaus, Phys. Rev. Lett. 105, 125501 (2010)

87. G. Domingues, S. Volz, K. Joulain, J.-J. Greffet, Phys. Rev. Lett. 94, 085901 (2005)

88. S. Xiong et al., Phys. Rev. Lett. 112, 114301 (2014)

89. V. Chiloyan, J. Garg, K. Esfarjani, G. Chen, Nat. Commun. 6, 6755 (2015)

90. I. Altfeder, A.A. Voevodin, A.K. Roy, Phys. Rev. Lett. 105, 166101 (2010)

91. K. Kloppstech et al., arXiv:1510.06311 (2015)

92. J. Schoenwald, E. Burstein, J. Elson, Solid State Commun. 12, 185 (1973)

93. E. Burstein, W.P. Chen, Y.J. Chen, A. Hartstein, J. Vacuum Sci. Technol. 11, 1004 (1974)

94. D. Tilley, Opt. Acta: Int. J. Opt. 30, 1501 (1983)

95. D.L. Mills, Phys. Rev. B 12, 4036 (1975)

96. T. Kume, T. Kitagawa, S. Hayashi, K. Yamamoto, Surf. Sci. 395, 23 (1998)

97. J.-P. Mulet, K. Joulain, R. Carminati, J.-J. Greffet, Appl. Phys. Lett. 78, 2931 (2001)

98. D.-Z.A. Chen, A. Narayanaswamy, G. Chen, Phys. Rev. B 72, 155435 (2005)

99. D.-Z.A. Chen, G. Chen, Appl. Phys. Lett. 91, 121906 (2007)

100. D.-Z.A. Chen, G. Chen, Frontiers in Heat and Mass Transfer 1, 023005 (2010)

101. G. Chen, Nanoscale Energy Transport and Conversion: A Parallel Treatment of Electrons, Molecules, Phonons, and Photons (Oxford University Press, Oxford, New York, 2005)

102. K. Fuchs, Math. Proc. Cambridge Philos. Soc. 34, 100 (1938)
103. H. Khosravi, D.R. Tilley, R. Loudon, J. Opt. Soc. Am. A 8, $112(1991)$

104. D.Z.A. Chen, A. Narayanaswamy, G. Chen, Proc. ASME Heat Transfer Division 376-2, 841 (2005)

105. R. Carminati, J.-J. Greffet, Phys. Rev. Lett. 82, 1660 (1999)

106. A. Narayanaswamy, G. Chen, Appl. Phys. Lett. 82, 3544 (2003)

107. J. Le Gall, M. Olivier, J.-J. Greffet, Phys. Rev. B 55, 10105 (1997)

108. C.A. Pfeiffer, E.N. Economou, K.L. Ngai, Phys. Rev. B 10, 3038 (1974)

109. P.-O. Chapuis, M. Laroche, S. Volz, J.-J. Greffet, Appl. Phys. Lett. 92, 201906 (2008)

110. J. Ordonez-Miranda et al., J. Appl. Phys. 115, 054311 (2014)

111. C. Thomsen, H.T. Grahn, H.J. Maris, J. Tauc, Phys. Rev. B 34, 4129 (1986)

112. V.E. Gusev, A.A. Karabutov, Laser Optoacoustics (AIP Press, New York, 1993)

113. A. Bartels et al., Rev. Sci. Instrum. 78, 035107 (2007)

114. P. Emma et al., Nat. Photon. 4, 641 (2010)

115. S. Schäfer, W. Liang, A.H. Zewail, J. Chem. Phys. 135 , 214201 (2011)

116. P. Yu, M. Cardona, Fundamentals of Semiconductors (Springer, Heidelberg, 1998)

117. H.J. Zeiger et al., Phys. Rev. B 45, 768 (1992)

118. R. Merlin, Solid State Commun. 102, 207 (1997)

119. T. Dekorsy, G. Cho, W. Kutt, H. Kurz, in Light Scattering in Solids VIII, edited by M. Cardona, G. Güntherodt (Springer, Berlin, 2000), Vol. 76

120. P. Ruello, V. Gusev, Ultrasonics 56, 21 (2015)

121. M. Perner et al., Phys. Rev. Lett. 85, 792 (2000)

122. V. Gusev, Opt. Commun. 94, 76 (1992)

123. J. Demsar, T. Dekorsy, in Optical Techniques for Solid-State Materials Characterization, edited by R.P. Prasankumar and J.T. Antoinette (CRC Press, Boca Raton, 2012)

124. G. Tas, H.J. Maris, Phys. Rev. B 49, 15046 (1994)

125. O.B. Wright, Phys. Rev. B 49, 9985 (1994)

126. O. Wright, V.E. Gusev, IEEE Trans. Ultrasonics, Ferroelectrics, Frequency Control 42, 331 (1995)

127. M. Lejman et al., J. Opt. Soc. Am. B 31, 282 (2014)

128. O. Matsuda et al., Phys. Rev. Lett. 93, 095501 (2004)

129. T. Pezeril et al., Phys. Rev. B 75, 174307 (2007)

130. A. Huynh et al., Phys. Rev. B 78, 233302 (2008)

131. M. Lejman et al., Nat. Commun. 5, 4301 (2014)

132. O.B. Wright, B. Perrin, O. Matsuda, V.E. Gusev, Phys. Rev. B 64, 081202 (2001)

133. P. Babilotte et al., Phys. Rev. B 81, 245207 (2010)

134. E.S.K. Young et al., Phys. Rev. B 86, 155207 (2012)

135. O.B. Wright, V.E. Gusev, Appl. Phys. Lett. 66, 1190 (1995)

136. G.C. Cho, W. Kütt, H. Kurz, Phys. Rev. Lett. 65, 764 (1990)

137. T. Pfeifer, T. Dekorsy, W. Kütt, H. Kurz, Appl. Phys. A 55, $482(1992)$

138. Y.-C. Wen et al., Phys. Rev. B 80, 195201 (2009)

139. P. Babilotte et al., Appl. Phys. Lett. 97, 174103 (2010)

140. A. Bartels, T. Dekorsy, H. Kurz, K. Köhler, Phys. Rev. Lett. 82, 1044 (1999)

141. O. Matsuda et al., Phys. Rev. B 71, 115330 (2005)

142. E. Péronne et al., Appl. Phys. Lett. 102, 043107 (2013) 
143. D. Lim et al., Phys. Rev. B 71, 134403 (2005)

144. C. v. Korff Schmising et al., Phys. Rev. B 78, 060404 (2008)

145. H. Wen et al., Phys. Rev. Lett. 110, 037601 (2013)

146. Y. Wang et al., Phys. Rev. B 88, 064307 (2013)

147. K. Norimatsu et al., Solid State Commun. 157, 58 (2013)

148. J. Flock, T. Dekorsy, O.V. Misochko, Appl. Phys. Lett. 105, 011902 (2014)

149. M. Weis et al., Phys. Rev. B 92, 014301 (2015)

150. M.V. Costache et al., Phys. Rev. Lett. 112, 086601 (2014)

151. D. Kim et al., Phys. Rev. Lett. 109, 166801 (2012)

152. A.V. Scherbakov et al., Phys. Rev. Lett. 105, 117204 (2010)

153. J.V. Jäger et al., Appl. Phys. Lett. 103, 032409 (2013)

154. A.J. Kent et al., Phys. Rev. Lett. 96, 215504 (2006)

155. C.L. Poyser, A.V. Akimov, R.P. Campion, A.J. Kent, Sci. Rep. 5, 8279 (2015)

156. A. Fainstein, N.D. Lanzillotti-Kimura, B. Jusserand, B. Perrin, Phys. Rev. Lett. 110, 037403 (2013)

157. I. Mahboob, K. Nishiguchi, A. Fujiwara, H. Yamaguchi, Phys. Rev. Lett. 110, 127202 (2013)

158. V. Gusev, P. Picart, D. Mounier, J.-M. Breteau, Opt. Commun. 204, 229 (2002)

159. Y. Yan, E.B. Gamble, K.A. Nelson, J. Chem. Phys. 83, $5391(1985)$

160. T. Kampfrath, K. Tanaka, K.A. Nelson, Nat. Photon. 7, $680(2013)$

161. R. Mankowsky et al., Phys. Rev. B 91, 094308 (2015)

162. R.J. Stoner et al., AIP Conf. Proc. 550, 468 (2001)

163. G.A. Antonelli, B. Perrin, B.C. Daly, D.G. Cahill, MRS Bull. 31, 607 (2006)

164. J.-F. Robillard, A. Devos, I. Roch-Jeune, P.A. Mante, Phys. Rev. B 78, 064302 (2008)

165. V. Juvé et al., Nano Lett. 10, 1853 (2010)

166. A. Amziane, L. Belliard, F. Decremps, B. Perrin, Phys. Rev. B 83, 014102 (2011)

167. C. Mechri, P. Ruello, V. Gusev, New J. Phys. 14, 023048 (2012)

168. F. Hudert, A. Bartels, T. Dekorsy, K. Khler, J. Appl. Phys. 104, 123509 (2008)

169. A.M. Lomonosov et al., ACS Nano 6, 1410 (2012)

170. C. Mechri et al., Appl. Phys. Lett. 95, 091907 (2009)

171. T. Dehoux, B. Audoin, J. Appl. Phys. 112, 124702 (2012)

172. C. Thomsen, H. Maris, J. Tauc, Thin Solid Films 154, 217 (1987)

173. M. Hettich et al., Appl. Phys. Lett. 98, 261908 (2011)

174. M.D. Losego et al., Nat. Mater. 11, 502 (2012)

175. A. Ayouch et al., ACS Nano 6, 10614 (2012)

176. I. Lisiecki et al., Nano Lett. 13, 4914 (2013)

177. A. Crut, P. Maioli, N. Del Fatti, F. Vallée, Phys. Rep. 549, 1 (2015)

178. F. Hudert et al., Phys. Rev. B 79, 201307 (2009)

179. S. Neogi, D. Donadio, Eur. Phys. J. B 88, 73 (2015)

180. G. Rozas et al., Phys. Rev. Lett. 102, 015502 (2009)

181. G.R.L.D. Landau, Phys. Z. Sowjet. 11, 18 (1937)

182. A. Akhieser, J. Phys. USSR 1, 277 (1939)

183. E. Chávez-Angel et al., Semicond. Sci. Technol. 29, 124010 (2014)

184. J.M. Ziman, Electrons and Phonons: The Theory of Transport Phenomena in Solids (Oxford University Press, Oxford, 1960)

185. A.D. O'Connell et al., Nature 464, 697 (2010)

186. M. Poot, H.S. van der Zant, Phys. Rep. 511, 273 (2012)
187. G. Kurizki et al., Proc. Natl. Acad. Sci. 112, 3866 (2015)

188. A.N. Cleland, Foundations of Nanomechanics (SpringerVerlag, Berlin, Heidelberg, New York, 2003)

189. A. Castellanos-Gomez, V. Singh, H.S.J. van der Zant, G.A. Steele, Annalen der Physik 527, 27 (2015)

190. W. Weaver, S.P. Timoshenko, D.H. Young, Vibration Problems in Engineering, 5th edn. (John Wiley \& Sons, Weinheim, 1990)

191. K.L. Ekinci, M.L. Roukes, Rev. Sci. Instrum. 76, 061101 (2005)

192. K. Ekinci, Small 1, 786 (2005)

193. D.V. Thourhout, J. Roels, Nat. Photon. 4, 211 (2010)

194. M. Imboden, P. Mohanty, Phys. Rep. 534, 89 (2014)

195. R. Lifshitz, M.C. Cross, in Review of Nonlinear Dynamics and Complexity, edited by H.G. Schuster (Wiley, 2008), Vol. 1, p. 1

196. Q.P. Unterreithmeier, E.M. Weig, J.P. Kotthaus, Nature 458, 1001 (2009)

197. S.S. Verbridge et al., J. Appl. Phys. 99, 124304 (2006)

198. Q.P. Unterreithmeier, T. Faust, J.P. Kotthaus, Phys. Rev. Lett. 105, 027205 (2010)

199. P.-L. Yu et al., Phys. Rev. Lett. 108, 083603 (2012)

200. P. Ovartchaiyapong et al., Appl. Phys. Lett. 101, 163505 (2012)

201. G.D. Cole et al., Appl. Phys. Lett. 104, 201908 (2014)

202. J. Chan et al., Appl. Phys. Lett. 101, 081115 (2012)

203. P.-L. Yu et al., Appl. Phys. Lett. 104, 023510 (2014)

204. D.T. Nguyen et al., Appl. Phys. Lett. 103, 241112 (2013)

205. T. Faust et al., Phys. Rev. Lett. 109, 037205 (2012)

206. T. Faust et al., Nat. Phys. 9, 485488 (2013)

207. H. Okamoto et al., Nat. Phys. 9, 480 (2013)

208. Y. Zhang et al., Phys. Rev. Lett. 113, 255502 (2014)

209. B.H. Schneider et al., Nat. Commun. 5, 5819 (2014)

210. R.B. Karabalin, M.C. Cross, M.L. Roukes, Phys. Rev. B 79, 165309 (2009)

211. H.J.R. Westra et al., Phys. Rev. Lett. 105, 117205 (2010)

212. I. Mahboob et al., Appl. Phys. Lett. 103, 153105 (2013)

213. M.H. Matheny et al., Nano Lett. 13, 1622 (2013)

214. Q.P. Unterreithmeier, T. Faust, J.P. Kotthaus, Phys. Rev. B 81, 241405 (2010)

215. A. Eichler et al., Nat. Nanotechnol. 6, 339 (2011)

216. R.B. Karabalin et al., Phys. Rev. Lett. 106, 094102 (2011)

217. M.H. Matheny et al., Phys. Rev. Lett. 112, 014101 (2014)

218. C.L. Degen et al., Proc. Natl. Acad. Sci. 106, 1313 (2009)

219. M. Poggio et al., Phys. Rev. Lett. 102, 087604 (2009)

220. J. Moser et al., Nat. Nanotechnol. 8, 493 (2013)

221. J. Chaste et al., Nat. Nanotechnol. 7, 301 (2012)

222. J. Bouchaud, IHS Technology, 2012, https:// technology.ihs.com

223. J. Arlett, E. Myers, M. Roukes, Nat. Nanotechnol. 6, 203 (2011)

224. K. Eom, H.S. Park, D.S. Yoon, T. Kwon, Phys. Rep. 503, $115(2011)$

225. L.Y. Gorelik et al., Phys. Rev. Lett. 80, 4526 (1998)

226. R.I. Shekhter et al., Nanoelectromech. Syst. 1, 1 (2013)

227. G.A. Steele et al., Science 325, 1103 (2009)

228. I. Yeo et al., Nat. Nanotechnol. 9, 106 (2014)

229. P. Ovartchaiyapong, K.W. Lee, B.A. Myers, A.C.B. Jayich, Nat. Commun. 5, 4429 (2014)

230. A. Barfuss et al., Nat. Phys. 11, 820 (2015)

231. T.J. Kippenberg, K.J. Vahala, Opt. Express 15, 17172 (2007) 
232. T.J. Kippenberg, K.J. Vahala, Science 321, 1172 (2008)

233. I. Favero, K. Karrai, Nat. Photon. 3, 201 (2009)

234. Cavity Optomechanics: Nano- and Micromechanical Resonators Interacting with Light, edited by $\mathrm{M}$. Aspelmeyer, T.J. Kippenberg, F. Marquardt (SpringerVerlag, Berlin, Heidelberg, 2014)

235. M. Eichenfield et al., Nature 462, 78 (2009)

236. J. Chan et al., Nature 478, 8992 (2011)

237. A.H. Safavi-Naeini et al., Phys. Rev. Lett. 108, 033602 (2012)

238. M. Maldovan, E.L. Thomas, Appl. Phys. Lett. 88, 251907 (2006)

239. J. Gomis-Bresco et al., Nat. Commun. 5, 4452 (2014)

240. A.H. Safavi-Naeini et al., Phys. Rev. Lett. 112, 153603 (2014)

241. G. Heinrich et al., Phys. Rev. Lett. 107, 043603 (2011)

242. V. Peano, C. Brendel, M. Schmidt, F. Marquardt, Phys. Rev. X 5, 031011 (2015)

243. O. Romero-Isart et al., Phys. Rev. A 83, 013803 (2011)

244. N. Kiesel et al., Proc. Natl. Acad. Sci. 110, 14180 (2013)

245. P. Mestres et al., Appl. Phys. Lett. 107, 151102 (2015)

246. J. Millen et al., Phys. Rev. Lett. 114, 123602 (2015)

247. J.D. Teufel, J.W. Harlow, C.A. Regal, K.W. Lehnert, Phys. Rev. Lett. 101, 197203 (2008)

248. J.D. Teufel et al., Nature 471, 204208 (2011)

249. F. Lecocq, J.D. Teufel, J. Aumentado, R.W. Simmonds, Nat. Phys. 11, 635639 (2015)

250. V. Singh et al., Nat. Nanotechnol. 9, 820 (2014)

251. T.J. Kippenberg et al., Phys. Rev. Lett. 95, 033901 (2005)

252. C. Metzger et al., Phys. Rev. Lett. 101, 133903 (2008)

253. C.H. Metzger, K. Karrai, Nature 432, 1002 (2004)

254. O. Arcizet et al., Nature 444, 71 (2006)

255. D. Kleckner, D. Bouwmeester, Nature 444, 75 (2006)

256. S. Gigan et al., Nature 444, 67 (2006)

257. A. Schliesser et al., Phys. Rev. Lett. 97, 243905 (2006)

258. F. Marquardt, J.P. Chen, A.A. Clerk, S.M. Girvin, Phys. Rev. Lett. 99, 093902 (2007)

259. I. Wilson-Rae, N. Nooshi, W. Zwerger, T.J. Kippenberg, Phys. Rev. Lett. 99, 093901 (2007)

260. J.D. Teufel et al., Nature 475, 359 (2011)

261. M. Aspelmeyer, S. Gröblacher, K. Hammerer, N. Kiesel, J. Opt. Soc. Am. B 27, A189 (2010)

262. P. Meystre, Annalen der Physik 525, 215 (2013)

263. T.A. Palomaki et al., Science 342, 710 (2013)

264. T.P. Purdy et al., Science 339, 801 (2013)

265. E.E. Wollman et al., Science 349, 952 (2015)

266. S. Weis, R. Riviere, S. Deleglise, E. Gavartin, O. Arcizet, A. Schliesser, T.J. Kippenberg, Science 330, 1520 (2010)

267. J.D. Thompson et al., Nature 452, 72 (2008)

268. J.C. Sankey et al., Nat. Phys. 6, 707 (2010)

269. S. Gröblacher, K. Hammerer, M.R. Vanner, M. Aspelmeyer, Nature 460, 724 (2009)

270. A. Nunnenkamp, K. Börkje, S.M. Girvin, Phys. Rev. Lett. 107, 063602 (2011)
271. D.J. Wilson et al., Nature 524, 325329 (2015)

272. M. Yuan, V. Singh, Y.M. Blanter, G.A. Steele, Nat. Commun. 6, 9491 (2015)

273. A. Pilan-Zanoni et al., arXiv:1508.01032 (2015)

274. A.G. Krause et al., Nat. Photon. 6, 768 (2012)

275. E. Gil-Santos et al., Nat. Nanotechnol. 10, 810816 (2015)

276. T.A. Palomaki et al., Nature 495, 210214 (2013)

277. J. Bochmann, A. Vainsencher, D.D. Awschalom, A.N. Cleland, Nat. Phys. 9, 712716 (2013)

278. T. Bagci et al., Nature 507, 8185 (2014)

279. C. Campbell, IEEE Proc. 77, 1453 (1989)

280. R. Aigner, in 2003 Topical Meeting on Silicon Monolithic Integrated Circuits in RF Systems, 2003. Digest of Papers (IEEE, Picataway, 2003), pp. 157-161

281. M.J. Hoskins, H. Morko, B.J. Hunsinger, Appl. Phys. Lett. 41, 332 (1982)

282. S. Büyükköse et al., Nanotechnol. 25, 135204 (2014)

283. D.M. Moss et al., Phys. Rev. Lett. 106, 066602 (2011)

284. M.V. Gustafsson et al., Science 346, 207 (2014)

285. A. Sologubenko, T. Lorenz, H. Ott, A. Freimuth, J. Low Temp. Phys. 147, 387 (2007)

286. G.E. Bauer, E. Saitoh, B.J. van Wees, Nat. Mater. 11, $391(2012)$

287. E. Pickwell-MacPherson, V.P. Wallace, Photodiagnosis and Photodynamic Therapy 6, 128 (2009)

288. P. Knobloch et al., Phys. Med. Biol. 47, 3875 (2002)

289. X.-C. Zhang, Phys. Med. Biol. 47, 3667 (2002)

290. Z. Popovic, E. Grossman, IEEE Trans. Terahertz Science and Technology 1, 133 (2011)

291. B.C. Daly et al., Appl. Phys. Lett. 84, 5180 (2004)

292. R. Li Voti, C. Sibilia, M. Bertolotti, Int. J. Thermophys. 26, 1833 (2005)

293. S.M. Nikitin et al., Sci. Rep. 5, 9352 (2015)

294. M. Mohseni, Y. Omar, G.S. Engel, M.B. Plenio, Quantum Effects in Biology (Cambridge University Press, New York, 2014)

295. S. Huelga, M. Plenio, Contemp. Phys. 54, 181 (2013)

296. E. Romero et al., Nat. Phys. 10, 676 (2014)

297. F.J. DiSalvo, Science 285, 703 (1999)

298. A. Majumdar, Science 303, 777 (2004)

299. B. Poudel et al., Science 320, 634 (2008)

300. L.-D. Zhao, V.P. Dravid, M.G. Kanatzidis, Energy Environm. Sci. 7, 251 (2014)

301. E. Chávez-Ángel et al., Appl. Phys. Lett. Mater. 2, 012113 (2014)

302. J. Cuffe et al., Phys. Rev. Lett. 110, 095503 (2013)

Open Access This is an open access article distributed under the terms of the Creative Commons Attribution License (http://creativecommons.org/licenses/by/4.0), which permits unrestricted use, distribution, and reproduction in any medium, provided the original work is properly cited. 